\title{
Multioctave high-dynamic range optical spectrometer for single-pulse, longitudinal characterization of ultrashort electron bunches
}

\author{
Omid Zarini@, ${ }^{1,2}$ Jurjen Couperus Cabadağ $\odot,{ }^{1}$ Yen-Yu Chang, ${ }^{1}$ Alexander Köhler@, ${ }^{1}$ \\ Thomas Kurz, ${ }^{1,2}$ Susanne Schöbel $\odot,{ }^{1,2}$ Wolfgang Seidel, ${ }^{1}$ Michael Bussmann $\odot,{ }^{1,3}$ \\ Ulrich Schramm $\odot^{1,2}$ Arie Irman $\odot^{1, *}$ and Alexander Debus $\odot^{1, \uparrow}$ \\ ${ }^{1}$ Helmholtz-Zentrum Dresden-Rossendorf, Bautzner Landstrasse 400, 01328 Dresden, Germany \\ ${ }^{2}$ Technische Universität Dresden, 01069 Dresden, Germany \\ ${ }^{3}$ Center for Advanced Systems Understanding, Untermarkt 20, 02826 Goerlitz, Germany
}

(Received 19 November 2020; revised 8 November 2021; accepted 12 November 2021; published 20 January 2022)

\begin{abstract}
We present design and realization of an ultrabroadband optical spectrometer capable of measuring the spectral intensity of multioctave-spanning light sources on a single-pulse basis with a dynamic range of up to eight orders of magnitude. The instrument is optimized for the characterization of the temporal structure of femtosecond long electron bunches by analyzing the emitted coherent transition radiation spectra. The spectrometer operates within the spectral range of $250 \mathrm{~nm}$ to $11.35 \mu \mathrm{m}$, corresponding to 5.5 optical octaves. This is achieved by dividing the signal beam into three spectral groups, each analyzed by a dedicated spectrometer and detector unit. The complete instrument was characterized with regard to wavelength, relative spectral sensitivity, and absolute photometric sensitivity, always accounting for the light polarization and comparing different calibration methods. Finally, the capability of the spectrometer is demonstrated with a coherent transition radiation measurement of a laser wakefield accelerated electron bunch, enabling to determine temporal pulse structures at unprecedented resolution.
\end{abstract}

DOI: 10.1103/PhysRevAccelBeams.25.012801

\section{INTRODUCTION}

Precise knowledge of the temporal structure of ultrashort electron bunches is key to understanding and control of modern linear accelerator driven light sources (LSs) [1] and high-peak current applications like beam-driven plasma wakefield accelerators [2-5]. On different energy scales, it is essential for applied research such as ultrafast probing of transient processes in matter by ultrafast electron diffraction [6,7]. In particular, the future refinement of laser wakefield accelerator (LWFA) [8] and related LS concepts [9-11] relies on improved diagnostic capabilities of the longitudinal phase space. It represents the main motivation of the current work as the temporal profile of wakefield accelerated bunches of a few femtosecond duration [12-15] with potential substructures [16-18] is closely linked to acceleration and injection conditions. Established bunch metrology techniques based on electro-optical sampling $[12,19,20]$ or the use of streak cameras [21] are mostly limited to sub-100 fs temporal resolution, whereas a newly

\footnotetext{
*A.Irman@hzdr.de

A.Debus@hzdr.de
}

Published by the American Physical Society under the terms of the Creative Commons Attribution 4.0 International license. Further distribution of this work must maintain attribution to the author(s) and the published article's title, journal citation, and DOI. established method based on transverse deflecting structures operated at X-band $\mathrm{rf}$ reached a resolution of better than $10 \mathrm{fs}[22,23]$. However, the latter requires a nontrivial jitter-free bunch-to-rf synchronization as well as a relatively high-rf power. Both requirements represent a challenge for multi-GeV electron beams at very low repetition rate as typically generated in the LWFA regime [24]. Though pulse-to-pulse stability in LWFAs has improved substantially in recent years, remaining fluctuations and in particular tracking changes require single-pulse availability of the complete information. Thus, in order to control beam quality and to improve the performance of plasmabased accelerators, the development of single-shot, highresolution spatiotemporal electron beam diagnostics is essential $[11,25]$.

The measurement of the spectral intensity of transition radiation (TR) emitted instantaneously when an electron bunch traverses a solid-vacuum interface [26] is a promising method to deduce the longitudinal bunch characteristics on the sub-femtosecond time scale [11,27]. In addition to its single-shot capability, the temporal resolution of this technique does not depend on the beam energy. Hence, it can be applied for $\mathrm{MeV}$ to $\mathrm{GeV}$ beams while the setup can be kept within a compact footprint. The TR spectrum contains information about the frequency dependent electron bunch form factor [28] from which the longitudinal charge distribution of the corresponding electron bunch can be reconstructed. The challenges linked 
to the approach lie in devising an absolutely calibrated multioctave spectrometer with high-spectral resolution and dynamic range, and in solving the nontrivial inverse problem of retrieving the temporal profile of the bunch from the measurement of spectral intensity alone. For the latter, advanced case-specific phase-retrieval algorithms for the reconstruction of the bunch have been developed $[18,29]$.

Here, we present all steps of the realization of an ultrabroadband optical spectrometer optimized to measure the TR spectrum over its full spectral bandwidth and with high resolution in single-pulse operation. This approach is essential for determining structural details and duration of an electron bunch in the range from 0.7 to 40 fs. Figure 1 presents the simulated spectral intensity of TR emitted by an electron bunch with a total charge of $20 \mathrm{pC}$ in a smooth, Gaussian distribution of $10 \mathrm{fs}$ full-width at half-maximum (FWHM) duration and $200 \mathrm{MeV}$ energy. The corresponding spectrometer range is from $250 \mathrm{~nm}$ to $11.35 \mu \mathrm{m}$. The radiation features two distinct spectral regions, the coherent part (CTR) and the incoherent part (ITR), separated by a transition range centered at a characteristic wavelength $\lambda_{\text {cutoff }}$ [28] of $3 \mu \mathrm{m}$. This wavelength corresponds to the bunch duration $\tau$ via $\tau \approx c \lambda_{\text {cutoff }}, c$ being the speed of the light. The intensity of the CTR scales up to $\propto N^{2}$ while ITR is proportional to $N$, when $N$ represents the number of electrons within the bunch. Hence, for $20 \mathrm{pC}$ of charge $\left(N \approx 10^{8}\right.$ electrons) as in this example an intensity difference of up to eight orders of magnitude is expected between the two regions. Beyond the illustrative example shown in Fig. 1, longitudinal electron bunch profiles in LWFA can be both diverse and complex, as these exhibit a broad range of bunch durations from fs to several $10 \mathrm{fs}$ $[12,13,15]$, as well as bunch charges from as small as $\mathrm{pC}$ to

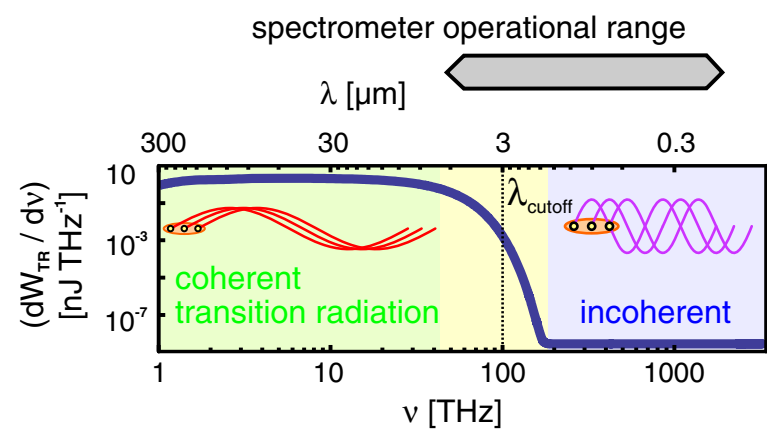

FIG. 1. Simulated spectral intensity of TR emitted by an unstructured, Gaussian-distributed electron bunch with $20 \mathrm{pC}$ charge, $200 \mathrm{MeV}$ energy and $10 \mathrm{fs}$ (FWHM) duration, as predicted by semianalytically integrating TR contributions over the 6D electron phase space according to Ref. [28]. Coherent transition radiation (CTR) is generated when the emitted wavelengths are longer than the bunch length, while incoherent transition radiation (ITR) dominates for shorter wavelengths. The operational range of the spectrometer is indicated, covering $250 \mathrm{~nm}$ to $11.35 \mu \mathrm{m}$. the nano-Coulomb-scale [30]. In addition, LWFA beams feature sub-structures down to the sub-fs scale [31] and bunch trains as in Ref. [32] require high resolution in the UV/VIS range to resolve resulting spectral modulations. This allows to detect and characterize sub-structures of ultrashort fs-scale-bunches, which previously were out of reach, providing novel insights to the injection and acceleration dynamics of laser plasma-based accelerators.

The minimal spectral resolution determines the uncertainty $\Delta \lambda / \lambda$ of a pulse duration measurement, resolving the spectral position of the coherent to incoherent transition, as well as the minimal resolvable spacing of a repetitive electron bunch signal $L=c T=\lambda^{2} / \Delta \lambda$ [32]. Particularly in the UV/VIS range, a high-spectral resolution allows to simultaneously resolve several bunches from multiple electron bunch injections within one plasma cavity within a plasma wavelength at a $20 \mu \mathrm{m}$-scale, to whole electron bunch trains across many consecutive acceleration cavities up to the mm scale.

From a more technical perspective, high-spectral resolutions in overlapping spectral regions of interest between spectrometer arms are useful for cross validation and calibration. In data analysis, spectral binning also allows to trade spectral resolution for increased S/N. Especially in the ultraviolet or visible (UV/VIS), where unstructured electron bunches tend to generate less coherent transition radiation (CTR), this allows to further extend the dynamic range towards detecting incoherent transition radiation.

Hence, measuring the absolute spectral intensity over the full spectral and dynamic range, i.e., the ITR, the transition, and the CTR ranges, is essential for extracting the TR spectral coherence and hence a meaningful reconstruction of the bunch distribution. This requires a spectrometer equipped with sensitive detectors supporting a high-dynamic range over the full spectral bandwidth. Simultaneously, a high-spectral resolution is mandatory for resolving spectral modulations arising from potential bunch sub-structures at sub-fs time scales.

Due to the limited spectral bandwidth of existing detector technologies and the limited dispersion and transmission properties of optical materials, the use of a modular spectrometer is indispensable. In the presented instrument, the input spectrum is divided into middle infrared (MIR), near infrared (NIR), as well as VIS to UV fractions by utilizing carefully selected beam splitters. Each fraction is thereafter spectrally decomposed using common yet range adapted dispersive elements such as prisms and gratings. The partial spectra are recorded by means of three detectors and, after post-processing, recombined into the extended final spectrum.

To account for any variation in the spectral response of individual detectors, as well as for transmission and reflection properties of all optical elements a full characterization of the instrument is performed over the entire extended spectral range. This includes a wavelength calibration [33-35], a relative spectral response calibration 
[36,37], and finally an absolute photometric calibration. The latter is the essential step for calculating the electron bunch form factor in terms of the absolute intensity of the TR for the electron bunch duration measurements.

The paper is organized as follows. In Sec. II design and setup of the spectrometer as well as a detailed review of spectrometer components are presented. In Sec. III, the full characterization of the spectrometer is described, presenting the three calibration steps. In Sec. IV, experimental results of TR measurements in LWFA experiments are shown, the data analysis described in Sec. V, and summarized in Sec. VI.

\section{DESIGN AND SETUP}

A schematic overview of the instrument with its three independent spectrometer arms and all central components is presented in Fig. 2. All optical components are installed inside a dedicated vacuum chamber for two reasons: First, this approach eliminates any absorption of the radiation of interest in air [38] due to vibrational-rotational transitions of molecules such as $\mathrm{H}_{2} \mathrm{O}$ or $\mathrm{CO}_{2}$ which fall into the range of 3 to $10 \mu \mathrm{m}$. Consequently, NIR and MIR detectors are directly attached to this vessel. The thickness of optical base plate and chamber walls have been optimized using the software Ansis to minimize deformations and thus to preserve sensitive spectrometer alignment after pumpdown. The maximum relative deformation of the base plate $(10 \mathrm{~cm}$

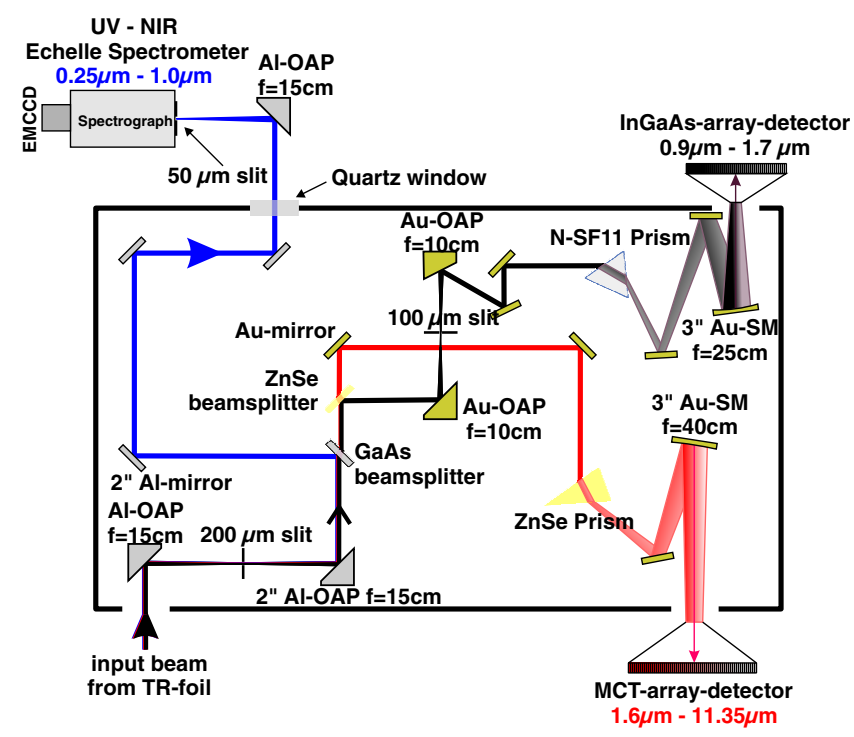

FIG. 2. Overview of the main components of the TR spectrometer showing a schematic top-view of the spectrometer design and highlighting the three main light paths. The vacuum chamber dimensions are $120 \mathrm{~cm} \times 68 \mathrm{~cm} \times 40 \mathrm{~cm}$. Additional optical elements such as polarizers, attenuators and bandpass filters, as well as imaging optics for monitoring the TR beam pointing used during the calibration and TR measurement are not shown here for clarity. Elements that have to be aligned during calibration or experimental runs are motorized. aluminum) and the walls have been simulated to amount to less than 0.1 and $0.3 \mathrm{~mm}$, respectively. Secondly, operation in vacuum enables the direct connection of the instrument to the accelerator vacuum, avoiding the use of any bandwidth-critical transmission element for TR beam transport.

In operation, a TR beam actively collimated close to its source enters the instrument and first passes a Keplerian telescope arrangement consisting of two $90^{\circ}$ off-axisparabolic mirrors (OAPs) with equal effective focal length $(\mathrm{EFL}=15 \mathrm{~cm})$. A slit is placed at the focal plane of the first OAP to set the overall spectral resolution of the spectrometer [39] (see Sec. II A). This OAP effectively images the TR source onto the slit. The recollimated TR beam is thereafter spectrally divided by two consecutive beam splitters (see Sec. II B). The first beam splitter, made of GaAs, reflects the UV to NIR region of the full TR spectrum, i.e., from 0.2 to $1.0 \mu \mathrm{m}$, which is analyzed by an Echelle spectrometer (see Sec. IID). The part of the spectrum transmitted through the GaAs is further divided by a $\mathrm{ZnSe}$ beam splitter into its MIR and NIR components. The reflected NIR fraction (0.9 to $1.7 \mu \mathrm{m})$ is passed through a second slit placed at the focal plane of a second telescope arrangement consisting of two gold coated OAPs. The recollimated beam is spectrally analyzed by a glass prism (N-SF11, see Sec. II C). The light is thereafter focused by a spherical mirror (SM) onto an InGaAs array detector (see Sec. II E 2). The remaining MIR fraction (1.6 to $12 \mu \mathrm{m}$ ) is directly sent to a ZnSe prism and focused by a SM onto a mercury cadmium telluride (MCT) array detector (see Sec. II E 3).

In the following, individual selection criteria for the components of the three spectrometer arms are discussed.

\section{A. Basics of a prism spectrometer}

The concept of a prism spectrometer is depicted in Fig. 3. A collimated light source LS is focused by lens $\mathrm{L}_{1}$ and illuminates an entrance slit $S_{1}$, placed in the focal plane (focal length $\mathrm{f}_{1}$ ) of $\mathrm{L}_{1}$. The beam is recollimated by $\mathrm{L}_{2}$ and passes through the prism $P$, where it is refracted by an angle $\delta(\lambda)$ depending on the wavelength $\lambda$, the incidence angle on the prism $\theta_{1}$ and its apex angle $\alpha$. The camera lens $\mathrm{L}_{3}$ images the entrance slit $S_{1}$ onto the detector array $S_{2}$, providing the wavelength dependent position signal $x(\lambda)$. The linear dispersion $d x / d \lambda$ of the spectrometer depends on the spectral dispersion $d n / d \lambda$ of the prism material and on the focal length $f_{3}$. Depending on the size of the detector array $\Delta x=x_{1}-x_{2}$, a spectral range $\Delta \lambda=\lambda_{1}\left(x_{1}\right)-\lambda_{2}\left(x_{2}\right)$ can be covered.

In the prism, the wavelength pair $\lambda$ and $\lambda+\Delta \lambda$ is split into beamlets with angular deviations $\delta$ and $\delta+\Delta \delta$. The angular separation is defined by the angular dispersion $d \delta / d \lambda$ (in units of $[\mathrm{rad} / \mathrm{nm}]$ ) as

$$
\Delta \delta=\left(\frac{d \delta}{d \lambda}\right) \Delta \lambda
$$




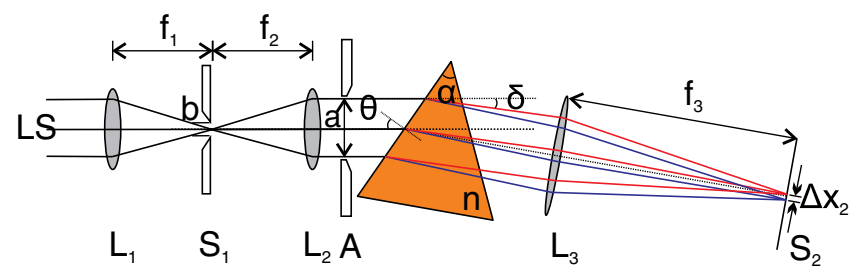

FIG. 3. Schematic of a prism spectrometer.

The camera lens then images the entrance slit $S_{1}$ onto the plane $S_{2}$. The distance $\Delta x_{2}$ between the two images $S_{2}(\lambda)$ and $S_{2}(\lambda+\Delta \lambda)$ is thus obtained by

$$
\Delta x_{2}=f_{3} \Delta \delta=f_{3} \frac{d \delta}{d \lambda} \Delta \lambda=\frac{d x}{d \lambda} \Delta \lambda
$$

where $d x / d \lambda$ denotes the linear dispersion of the spectrometer (in units of $[\mathrm{mm} / \mathrm{nm}]$ ).

The spectral resolution is assumed to be dominated by diffraction depending on the aperture $a$ and focal length $f_{3}$ (see Fig. 4). According to the Rayleigh criterion [40] the minimum distance between two resolvable slit images reads as [38]

$$
\Delta x_{R}=f_{3}(\lambda / a)
$$

Further taking into account the finite size of the slit $b$ and applying geometrical optics, the width of the image of the slit is obtained by

$$
\Delta x_{b}=b\left(f_{3} / f_{2}\right) .
$$

The separation $\Delta x_{2}$ between the peaks of the two image distributions $I_{1}\left(\lambda_{1}\right)$ and $I_{2}\left(\lambda_{2}\right)$ must exceed the sum of the above (see Fig. 4)

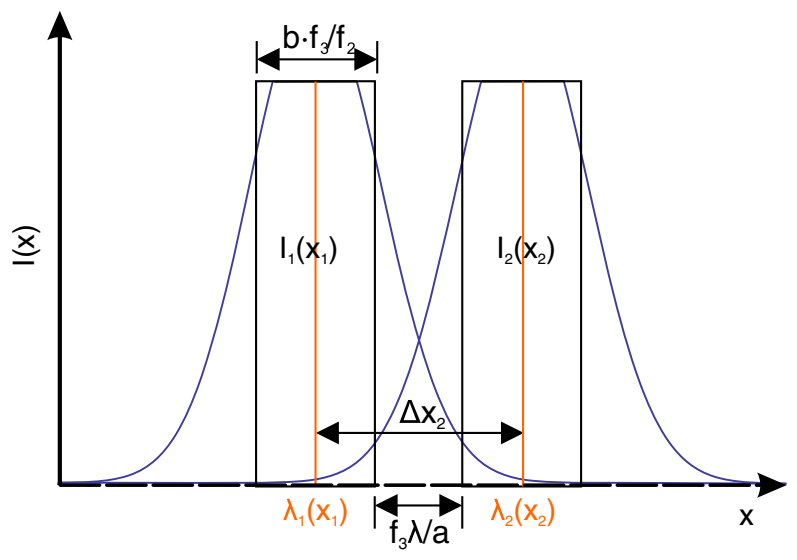

FIG. 4. Illustration of the resolution limit of two closely overlapping spectral lines, where $x$ denotes a position on the spectrometer detector and corresponds to some wavelength $\lambda(x)$.

$$
\Delta x_{2} \geq f_{3} \frac{\lambda}{a}+b \frac{f_{3}}{f_{2}}
$$

The smallest resolvable wavelength interval $\Delta \lambda$, the spectral resolution, is obtained from Eq. (2) as

$$
\Delta \lambda \geq\left(\frac{\lambda}{a}+\frac{b}{f_{2}}\right)\left(\frac{d \delta}{d \lambda}\right)^{-1}
$$

Note, that for an infinitely small entrance slit $b$ the spectral resolution is dominated by the diffraction caused by the much larger aperture $a$ [Eq. (3)], typically given by the size of the beam optics. Thus, optimum slit width settings can be chosen to be compliant with transmission efficiency optimization of the apparatus.

Finally, the spectral resolving power $R$ is derived from the spectral resolution to

$$
\begin{aligned}
R & =\left|\frac{\lambda}{\Delta \lambda}\right|=\lambda\left(\frac{\lambda}{a}+\frac{b}{f_{2}}\right)^{-1}\left(\frac{d \delta}{d \lambda}\right) \\
& =\lambda\left(\frac{\lambda}{a}+\frac{b}{f_{2}}\right)^{-1} f_{3}^{-1}\left(\frac{d x}{d \lambda}\right) .
\end{aligned}
$$

\section{B. Beam splitters}

Dichroic beam splitters are normally designed for a specific spectral bandwidth either in the VIS or IR range and thus not applicable for the separation of broad spectra. By using uncoated infrared transparent bulk material and taking advantage of its Fresnel reflection and transmission properties, two consecutive beam splitters are selected in order to spectrally separate the input beam into three spectral bands (see Fig. 2). For the first beam splitter, a $6 \mathrm{~mm}$ thick Gallium-Arsenide-plate (GaAs) with $12 \mathrm{~min}$ wedge angle is used to separate the UV-NIR range of the spectrum $(250 \mathrm{nn}$ to $1.0 \mu \mathrm{m})$ from the TR beam. The small remaining fraction of Fresnel reflected longer wavelength light is further attenuated by the fused silica exit window (Corning 7980).

For the NIR and MIR Zinc-Selenide (ZnSe) [41] is the preferred material for transmission optics because of its low absorptivity at infrared wavelengths $\left(\leq 0.0005 \mathrm{~cm}^{-1} @\right.$ $10.6 \mu \mathrm{m})$ and its acceptable visible light transmission for alignment purposes. For the second beam splitter, a $3 \mathrm{~mm}$ thick $\mathrm{ZnSe}$ plate with 6 min wedge angle is utilized.

Transmission and reflection parameters are calculated by means of Fresnel equations [42] with respect to s- and p-polarization (in the spectrometer plane). The wavelength dependent refractive indices of these materials are modeled applying Sellmeier-polynomials [43]. The absorption as well as losses from secondary internal reflections in the GaAs material is taken into account while absorption in $\mathrm{ZnSe}$ bulk material is negligible. The results of this calculation are shown in Fig. 5. 


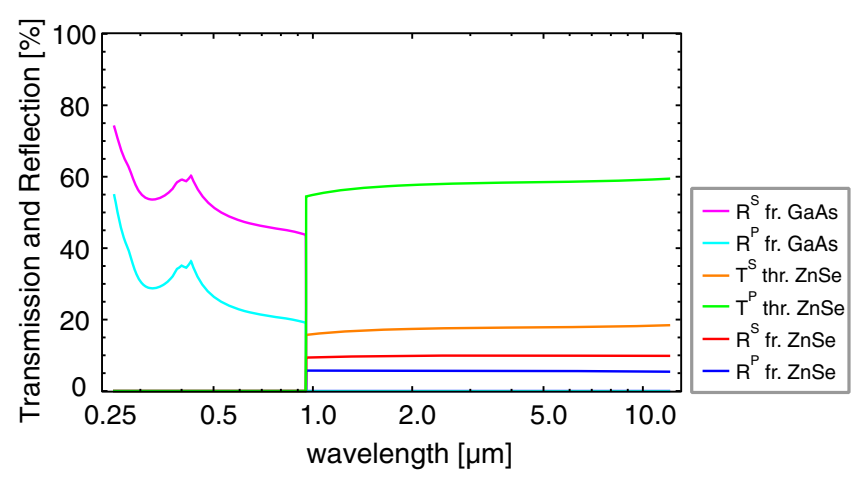

FIG. 5. Input beam transmission and reflection for the GaAs and $\mathrm{ZnSe}$ beam splitters. GaAs, with a sharp transmission edge at around $900 \mathrm{~nm}$ and low-bulk absorption of $\leq 0.01 \mathrm{~cm}^{-1} @$ $10.6 \mu \mathrm{m}$, is ideal for a spectral separation of the UV-NIR range from the near to the MIR range. ZnSe reflects on average $7 \%$ of the beam and transmits $18 \%$ and $60 \%$ of the input beam for s- and p-polarization, respectively.

\section{Prism optimization}

The free spectral range of a spectrometer $F_{\lambda}$ is defined as the wavelength range for which an unambiguous relationship between $\lambda$ and the position $x(\lambda)$ at the detector surface exists. A single prism-based instrument covers a broad spectral range depending on its dispersion $n(\lambda)$ at a resolution that is rather low compared to grating-based solutions. For the latter, $F_{\lambda}$ is in contrast limited by overlapping diffraction orders. Furthermore, prisms exhibit higher throughput and lower stray light characteristics. For customizing the geometry of a prism and selecting its material, the following optimization criteria are applied: (i) The free spectral range $F_{\lambda}=\lambda_{\text {high }}-\lambda_{\text {low }}$ is set with respect to the spectral response of available detectors, including transmission and reflection properties of the optics. Due to a good quantum efficiency (QE) of the InGaAs detector (see Sec. II E 2) in the range of $800 \mathrm{~nm}$ to $1.7 \mu \mathrm{m}$ (limits@20\% level), and the cutoff of the GaAs beam splitter at $900 \mathrm{~nm}$ (see Sec. II B) the spectral range for the NIR arm is set from $900 \mathrm{~nm}$ to $1.7 \mu \mathrm{m}$. According to the spectral response of the MCT detector (see Sec. II E 3) the spectral range of 1.6 to $11.35 \mu \mathrm{m}$ is defined for the MIR arm including some spectral overlap of $100 \mathrm{~nm}$ between NIR and MIR ranges, facilitating the later absolute cross calibration of the spectrometer arms. (ii) The linear dispersion $d x / d \lambda$ introduced by the prism should cover the entire detector array with regard to the focal length of the focusing optic. Here, SMs are utilized in order to minimize imaging errors across the spectrum. As the SM is irradiated at a small off-axis angle (in our case $\sim 10^{\circ}$ ), the choice causes a slight astigmatism leading to elliptical foci on the detector array plane. As these foci are smaller than the size of the InGaAs pixels $(500 \times 25 \mu \mathrm{m})$ no intensity loss is expected. (iii) The prism should feature linearity in dispersion for achieving a uniform spectral

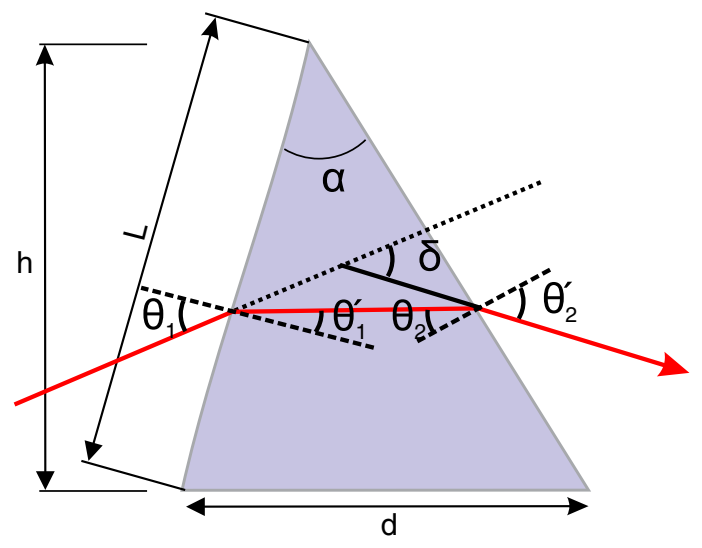

FIG. 6. Ray trace through a prism. $\theta_{1}, \theta_{1}^{\prime}, \theta_{2}, \theta_{2}^{\prime}$ are the incident and refractive angles at the first and second prism interface, respectively, $\alpha$ the prism apex angle, $d, L, h$ are the geometric dimensions of the prism, and $\delta$ is the deflection angle with respect to the incident beam. Note the sign convention of the angles $\theta$ and $\delta$, in which clockwise angles with respect to the surface normal have negative sign and vice versa.

resolution on the corresponding detector array. The nonlinearity (NL) of the dispersion is defined by the spectral sampling ratio (SSR)

$$
\operatorname{SSR}=\max \left|\frac{\mathrm{d} \delta}{\mathrm{d} \lambda}\right| / \min \left|\frac{\mathrm{d} \delta}{\mathrm{d} \lambda}\right|,
$$

representing a good figure of merit for the optimization of the prism geometry. (iv) Large angles $\left(\theta_{1}\right.$ and $\theta_{2}^{\prime}$ in Fig. 6) on the prism cause large projected beam diameters requiring large prism optics, as well as precise angular alignment. Additionally, these scenarios lead to significant losses due to reflection on the prism surfaces. For excluding such grazing incidence designs the constraints $\theta_{1}<60^{\circ}$ and $\theta_{2}^{\prime}<60^{\circ}$ are assumed for prism optimization. (v) Finally, the prism geometry needs to be chosen such that its refractive surfaces are compliant with the beam diameter and the overall size remains reasonable.

The prism geometry is modeled in Mathematica [44] following geometrical optics (see Fig. 6) and ray paths are calculated with the help of Snell's law. The deflection angle of a beam behind the prism can be expressed as [45]

$$
\begin{aligned}
\delta\left(\lambda, \theta_{1}, \alpha\right)= & -\alpha+\theta_{1} \\
& +\arcsin \left(n(\lambda) \sin \left(\alpha-\arcsin \left(\frac{\sin \left(\theta_{1}\right)}{n(\lambda)}\right)\right)\right) .
\end{aligned}
$$

A comprehensive description of the optimization criteria can be found in Refs. [45-47]. A global optimization algorithm based on the differential evolution method was employed in order to determine the prism geometry for a series of available infrared materials. The selected prism designs, N-SF11 [48] for the NIR range and ZnSe [49] for 

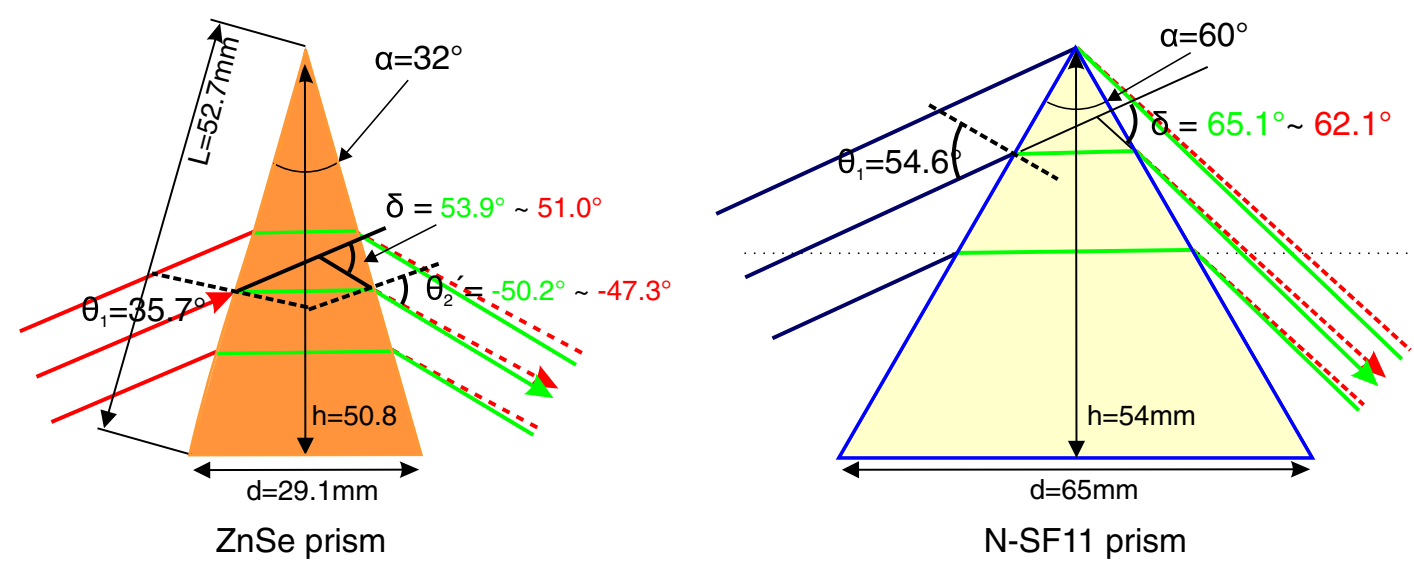

FIG. 7. Illustration of N-SF11 and ZnSe prism geometries, designed for NIR (0.9 to $1.7 \mu \mathrm{m})$ and MIR (1.6 to $12 \mu \mathrm{m})$ ranges of the spectrometer, respectively.

the MIR range, are documented in Fig. 7. In principle, the spectral linearity of the prism spectrometer could be improved by a double prism design, in particular by the combination of $\mathrm{ZnSe}$ and $\mathrm{NaCl}$ prisms. As it turned out the remaining gap between the prisms, due to the lack of an interfacing glue material suitable for the required spectral range, leads to multiple reflections. Furthermore the hygroscopic property of $\mathrm{NaCl}$ is disadvantageous. Thus this option was discarded for the MIR arm.

\section{Echelle spectrometer}

An echelle spectrometer supports unprecedented bandwidth in single pulse operation with high resolution and spectral sensitivity by combining a prism with a grating subsequently acting in orthogonal dispersion planes. The echelle grating diffracts the spectrum into several orders, each of which represents a portion of the high-resolution spectrum. The prism then enables order separation when imaged onto a 2D detector array. A commercial echelle spectrometer ME5000 [50] is implemented in the TR spectrometer for the analysis of the UV-NIR range (200 to $1030 \mathrm{~nm}$ ). The input beam is focused through a $50 \mu \mathrm{m}$ slit at the entrance of the echelle spectrograph, then recollimated by a F/7 SM and subsequently refracted by a double prism. The dispersed beam in turn falls onto a grating and is thereby diffracted perpendicularly to the refraction plane of the prism. A focusing mirror images the entrance slit onto a 2D EMCCD detector (see Sec. II E 1). The grating has a groove density of $52.13 \mathrm{~mm}^{-1}$ and is used at high-grating orders, i.e., 20 to 100 and blazed under $32.35^{\circ}$.

The free spectral range for a single order $m$ is given by

$$
F_{\lambda}^{m}=\frac{\lambda_{m}^{o}}{m\left(1-(2 m)^{-2}\right)},
$$

where $\lambda_{m}^{0}$ denotes the central wavelength of this order. The overall bandwidth thus is the sum of the number of orders that can be covered by the $2 \mathrm{D}$ detector chip. The efficiency of the echelle spectrometer depends mainly on the efficiency of its grating and the QE of the coupled detector. Although its grating deflection angle $\phi$ is not varying much over a certain spectral order $\left(\leq 2^{\circ}\right)$ [51,52], the reflection coefficient of the grating and therefore the grating efficiency considerably drops from its maximum in the middle of each order toward the edges of each spectral order to about half of its value [see Fig. 8(c)]. The strong variations in the spectral efficiency of the echelle spectrometer over its entire spectral range is illustrated in Fig. 8, where a QuartzTungsten-Halogen (QTH) lamp is used as broad calibration source.

In the 2D CCD raw data, each spectral order approximately follows a straight line with some fixed width. Thus for data analysis, we extract the center line of maximum intensity for each order and sum up the signal over the line width. At this stage, the resulting 1D signal arrays of all orders are still spectrally overlapping and have no well defined end points. After wavelength calibration using a spectral line source, the spectral endpoints of each order are determined by wavelength position, at which either the pre- or proceeding order becomes spectrally more sensitive. All these such wavelength calibrated and truncated order-spectra are then joined to a single spectrum, see Fig. 8(c).

\section{E. Detectors}

In order to record information over the broad spectral range of the instrument at high resolution and high sensitivity simultaneously we utilize an MCT array detector (1.6 to $11.35 \mu \mathrm{m})$, an InGaAs array detector $(0.9 \mu \mathrm{m}$ to $1.7 \mu \mathrm{m})$, and an EMCCD detector $(250 \mathrm{~nm}$ to $1.0 \mu \mathrm{m})$ as detailed in the following.

\section{EMCCD detector}

The 2D spectrum of the echelle spectrograph (see Sec. II D) is recorded with a 14-bit, back illuminated electron multiplying charged coupled devices (EMCCD) 

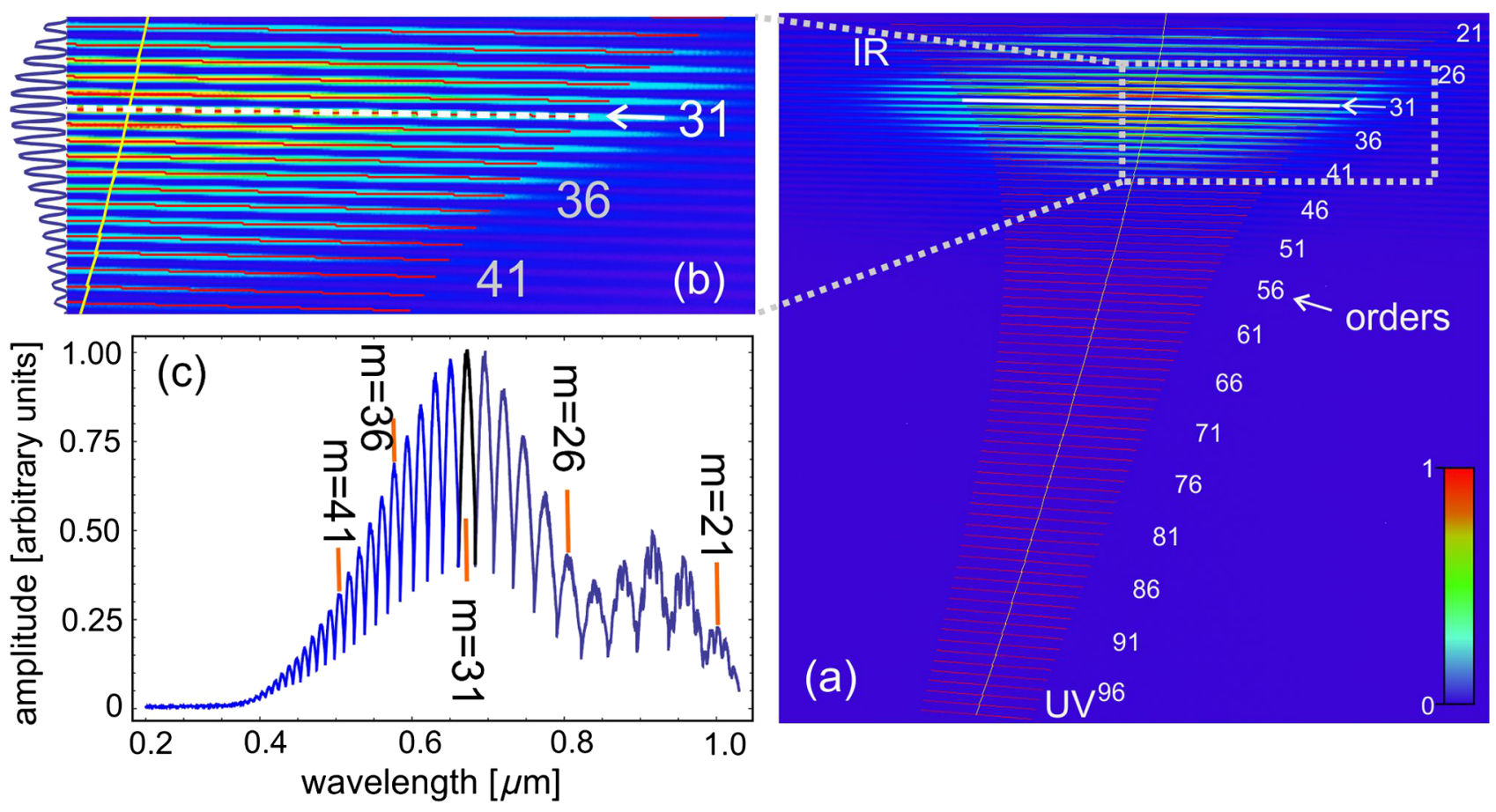

FIG. 8. Sample measurement of the UV-VIS spectrum using a QTH lamp. (a) illustrates the full image acquired with the 2D detector of the echelle spectrometer. The horizontal red lines indicate the (numbered) diffraction orders. The orange line connects the central wavelength $\lambda_{m}^{0}$ of each order. (b) shows a zoomed part of (a) including a vertical line out showing the separation of the diffraction orders. The vertical extension of individual orders covers around 10 pixels. (c) presents a complete spectrum sampled from all orders as detailed in Sec. II D and III A 1.

detector [53]. Its active area consists of $1024 \times 1024$ pixels with a pixel size of $13 \times 13 \mu \mathrm{m}$. The EMCCD sensor technology enables the measurement of very weak signals by suppressing the readout noise during the readout process. This is a trade-off reducing the camera's effective dynamic range as the feature is realized by amplifying the signal in electron multiplier (EM) registers before it is transferred to the output amplifier and A/D-converter. By suppressing the readout noise using an EM gain of 30, an effective dynamic range larger than 14 bit could be maintained. The detector head is enclosed in a vacuum container behind an AR coated fused silica window. Furthermore a built-in thermocouple cools the chip down to $-95^{\circ} \mathrm{C}$ reducing the (thermal) dark current in the detector by more than one order of magnitude.

\section{InGaAs detector}

For the NIR detection in the range of $0.9-1.7 \mu \mathrm{m}$ with high QE, minimized noise, and a high dynamic bandwidth, an InGaAs array-detector [54] is used. The detector array consists of 512 pixels, each $25 \mu \mathrm{m}$ wide and $500 \mu \mathrm{m}$ high. As the EMCCD the detector array is mounted in a dedicated vacuum invironment, cooled to $-90^{\circ} \mathrm{C}$, and accessed through an uncoated fused silica window.

\section{MCT detector}

In order to measure the spectrum in the MIR range from 1.6 to $11.35 \mu \mathrm{m}$, a photoconductive MCT array detector [55] is applied. The semiconductor-based MCT detector is more sensitive and faster than thermal (pyroelectric) detectors featuring an orders of magnitudes higher peak specific detectivity $D^{\star}=2 \times 10^{10} \mathrm{~cm} \mathrm{~Hz}^{1 / 2} / \mathrm{W}$ $[56,57]$. Peak response was customized by the manufacturer from 2 to $14 \mu \mathrm{m}$ (@ 20\% spectral response) by adjusting the alloy composition of its ternary compound. The MCT detector array consists of 64 pixels. The active area of each pixel is $300 \mu \mathrm{m}$ wide and $600 \mu \mathrm{m}$ high. An additional inactive space of $25 \mu \mathrm{m}$ separates the pixels from each other. The chip is housed in a Dewar flask behind an AR-coated $\mathrm{ZnSe}$ window and cooled with liquid nitrogen $\mathrm{LN}_{2}$ to $74 \mathrm{~K}$. The integration time window for recording can be set in $10 \mathrm{~ns}$ steps from 54 to $2614 \mathrm{~ns}$. It should be noted that the MCT electronics measure changes in resistance. The readout amplifier thus uses as reference the average bias current from the $30 \mu$ s preceding the integration time window for data acquisition. This measurement method is well suited for signals which occur within a ns- $\mu$ s integration time. However, in order to perform a decent measurement regarding the absolute energy of a signal, the incidence pulse must be shorter than the time constant of the detector, i.e., $<400 \mathrm{~ns}$.

\section{CALIBRATION}

In the following, the calibration refers to all radiation passing through the $200 \mu \mathrm{m}$ input slit of the spectrometer 
within its acceptance half angle of the collection optics, which is $16 \mathrm{mrad}$ in the setup presented here, see Fig. 2.

The complex procedure providing absolute calibration of spectral sensitivity of the full instrument, in units of measured detector signal per energy per spectral bandwidth $[($ counts or $\mathrm{V}) \times \mu \mathrm{m} / \mathrm{J}]$, is divided into three separate tasks. Following wavelength calibration, a relative sensitivity calibration is performed. For selected wavelengths, a final calibration based on photometric measurements transforms the relative calibration into an absolute. All steps include transmission and reflection properties of all optical elements as well as the spectral response and QE of the individual detectors and distinguish between s- and ppolarization of the incoming light. Consequently, a wide variety of calibration sources is used. With an exception for the echelle spectrometer all calibration sources are coupled into the spectrometer through its entrance. An overview of the calibration sources is depicted in Fig. 9. These include monochromatic laser sources, calibrated spectral emission lines sources, characterized absorption foils and bandpass filters (BPFs), as well as calibrated continuous sources such as black body (BB), QTH, and Deuterium lamps. The wavelength calibration in the MIR range is performed by means of absorption lines of plastic films ( $\sim 10 \mu$ m thick) back illuminated by a BB reference spectrum. Spectral emission from an Argon source (Ar) and a Mercury-Argon source $(\mathrm{HgAr})$ is used in the NIR and UV-VIS ranges, respectively. For the relative calibration photometrically defined broadband sources are employed, yet, in order to minimize errors, only the shape of the respective spectrum is exploited and not the photometric calibration. The absolute calibration is instead performed via a number of different continuous-wave $(\mathrm{CW})$ lasers at selected wavelengths.

The wavelength calibration is discussed in Sec. III A where the results are subsequently presented for each spectrometer arm. The relative as well as absolute calibration procedures follow in Secs. III C and III D.

The goal of the calibration is to provide the spectral sensitivity of the full instrument in units of measured detector signal per energy per spectral bandwidth $[($ counts or $\mathrm{V}) \times \mu \mathrm{m} / \mathrm{J}]$ and, as well as the spectral NEE in units of energy per spectral bandwidth $[\mathrm{J} / \mu \mathrm{m}]$ for estimating signal to noise ratios (SNRs) and minimal detectable signals. Before IR calibration measurements are started, the TR spectrometer chamber is purged by dried nitrogen over several hours. This removes, in particular, moisture and creates a much drier calibration environment and minimizes IR absorption.

\section{A. Wavelength calibration}

The wavelength calibration of a spectrometer involves the measurement of spectral lines at well-known wavelengths from the emission of calibration light sources (LSs) [34] or absorption lines of a known absorber material. The relationship between the wavelength and the corresponding pixel positions on the detector is obtained by a polynomial fit [35]. In the following, we discuss a specific protocol for each of the spectrometer arms separately.

\section{Echelle spectrometer}

A HgAr LS emits first-order mercury and argon lines ranging from 253 to $922 \mathrm{~nm}$ and second-order argon lines up to $1700 \mathrm{~nm}$. A $50 \mu \mathrm{m}$ diameter fiber optic [58] is used to couple the source directly into the echelle spectrometer. Identification of these spectral lines on the EMCCDdetector is performed by the wavelength calibration procedure of the manufacturer acquisition software [50]. Following the search of the relevant spectral lines, the boundaries of each diffraction order with respect to their central wavelengths $\lambda_{m}^{0}$ are thereby determined. Since the spectral bandwidth of any order is small compared to its entire spectral range, linear interpolation on each order is performed to accomplish the wavelength calibration of individual diffraction orders. The overall calibration is then carried out by combining the diffraction orders and performing a fifth-order polynomial fit. The calibration curve addresses the central pixel for each wavelength from which \pm 5 pixels in the corresponding column, perpendicular to the orders, have to be summed up in order to read out the total spectral intensity (see Sec. III D). As presented in Fig. 10, while the spectral bandwidth of

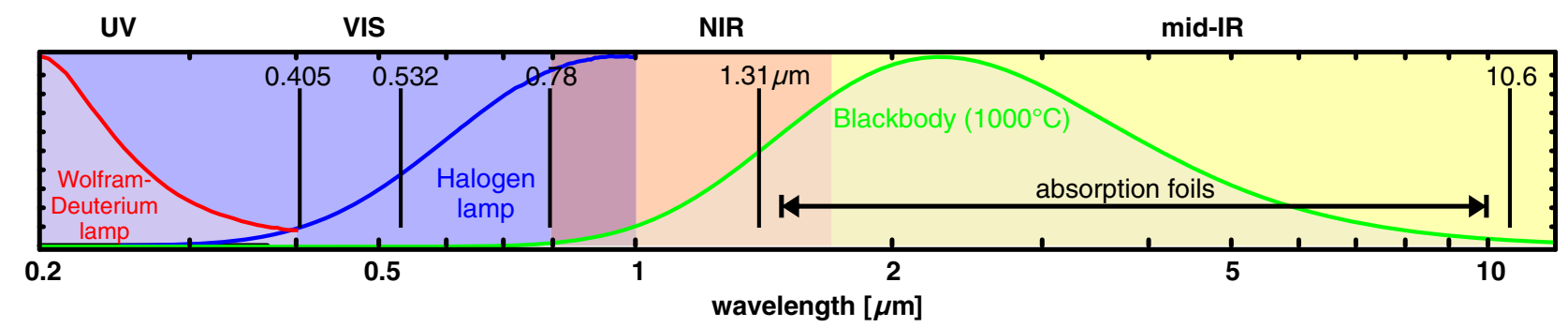

FIG. 9. Overview of the light sources used for the different steps of the calibration procedure comprising continuous light sources (LSs), laser lines (black lines), and a set of absorption foils (TPX, PP, HDPE, and Mylar). The spectra of the HgAr and Ar spectral lamps are not shown here. 


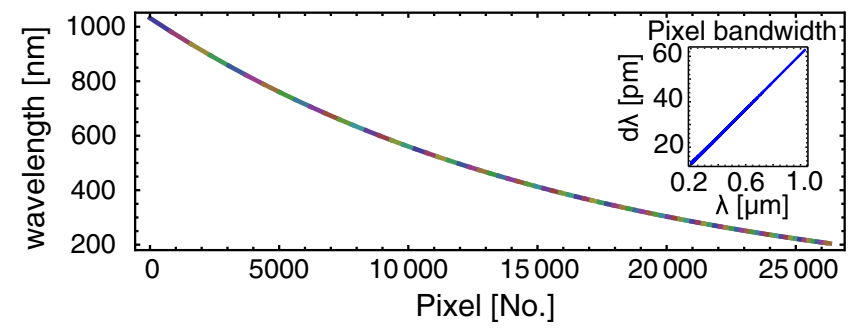

FIG. 10. Wavelength calibration fit for the echelle spectrometer: Each color-distinguished partition of the curve represents a diffraction order. The inset here and in the following figures shows the spectral bandwidth covered by individual pixels as a function of wavelength.

each order is decreasing toward shorter wavelengths, the resolution is increasing with the order number.

Our data analysis of the CCD raw data generally follows a procedure similar to the manufacturers data acquisition software. However, we improved the data extraction of the individual spectral orders, as the endpoints of each order-line were not always stable over time (temperature dependence), in practice drifting by as much as three pixels vertically. While this usually is a negligible error in wavelength, it is relevant for the relative and absolute photometric calibration described later, which requires to capture the full signal. Failing to account for this drift immediately generates distinct artifacts in the final calibrated signal, that show as characteristic variations of the echelle efficiency pattern being imprinted on the measured signal.

This is solved by fine-tuning the region of interests in data extraction, which for each order is defined by two endpoints determined during calibration and a line height of five pixels. For any measured dataset, we thus vary the vertical positions of each spectral endpoint by up to \pm 3 pixels and compare the spectrally and vertically integrated raw signals, optimizing for the configuration with maximum signal. These resulting corrections change only gradually over time and order number and essentially ensure that the full height of the respective spectral orders is extracted.

\section{InGaAs detector}

The alignment of the NIR arm is performed by means of a single mode polarization-maintaining fiber optic coupled laser of central wavelength $\lambda=1.31 \mu \mathrm{m}$ and $\Delta \lambda=10 \mathrm{~nm}$ bandwidth [59]. The central pixel value is used as the first calibration point and later reused for the absolute calibration (see Sec. III D. Further calibration is based on a lowpressure Ar source [60] emitting lines between $700 \mathrm{~nm}$ and $1.7 \mu \mathrm{m}$. The Ar emission is transported via fiber optic [58] and collimated by an OAP in order to transport it using folding mirrors into the spectrometer chamber. Additionally, the transmission signal of two BPFs at $950 \mathrm{~nm}$ and $1.65 \mu \mathrm{m}$ is included. Over all calibration

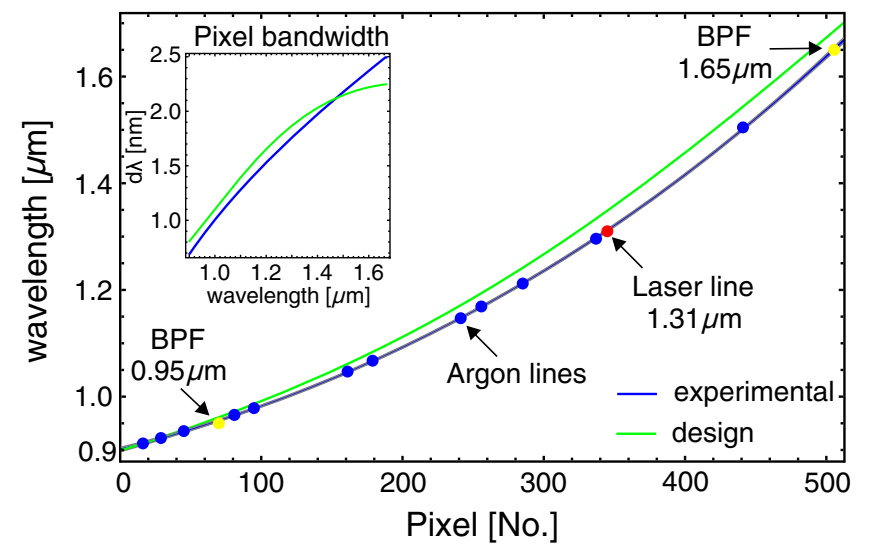

FIG. 11. Wavelength calibration of the InGaAs detector: Several Ar lines are identified (marked in blue) on the InGaAs array detector. The marks for the BPF's and the alignment laser fall well in line. The blue solid line represents a third order polynomial fit. The $3 \sigma$ standard deviation of the fit model is found to be on average $0.34 \%$.

points a three-order polynomial fit is performed as shown in Fig. 11.

\section{MCT detector}

The wavelength calibration of the MCT detector is performed using absorption lines of four different thin $(\sim 10 \mu \mathrm{m})$ Teflon films (TPX, HDPE, PP, Mylar) whose transmission spectra had been characterized over the spectral range of interest for the MCT detector beforehand by using a Fourier transform infrared (FTIR) spectrometer ( $4 \mathrm{~cm}^{-1}$ resolution). These absorption lines are then measured with the MCT detector using the $\mathrm{BB}$ radiator as the reference LS. Over the calibration lines including a $\mathrm{CO}_{2}$ laser line and two BPFs at 1.65 and $2 \mu \mathrm{m}$ a third-order polynomial is fitted.

The result of the MCT calibration, presented in Fig. 12, indicates that the different absorption foils and BPFs provide a variety of calibration points which enable a relative error of only $4 \%$ on average.

\section{B. Resolution and resolving power}

The theoretical and experimental optical resolution of each individual spectrometer arm is evaluated. Values for the theoretical angular dispersion $d \delta / d \lambda$ are obtained from Eq. (9) and the experimental linear dispersion $d x / d \lambda$ follows from the wavelength fits. The latter are evaluated on a discrete grid representing the dimension of the active area of the corresponding detectors. Figure 13 depicts the spectral resolution for each of the spectrometer arms. The spectral resolution increases from the MIR range to the UV range by more than three orders of magnitude, well matching the experimental signatures expected for frequency modulated broadband CTR spectra (see Sec. IV). 


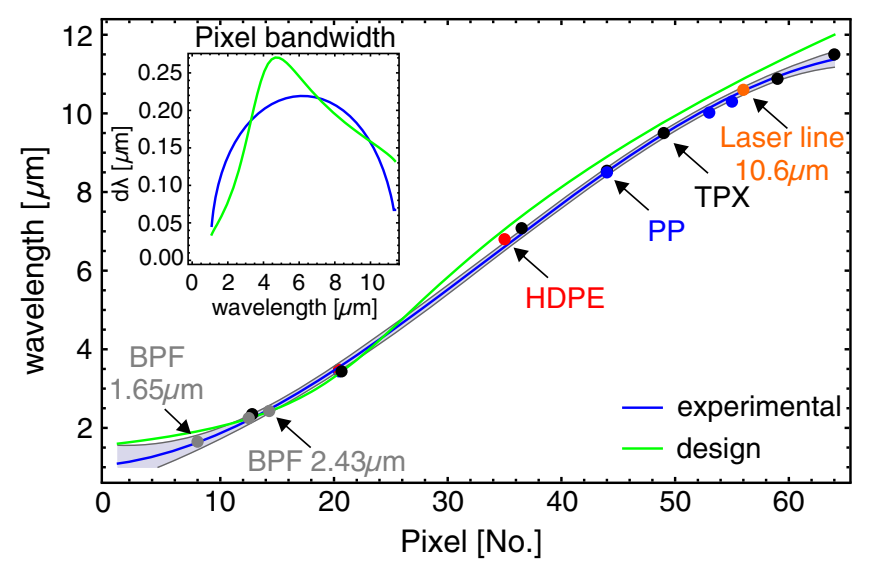

FIG. 12. Wavelength calibration of the MCT detector: The blue solid line indicates a third-order polynomial fit, with the light blue band representing the $3 \sigma$ confidence interval of the fit.

Although the smallest detectable wavelength of the echelle spectrometer amounts to $200 \mathrm{~nm}$, the low throughput of the spectrometer below $250 \mathrm{~nm}$ prevents an accurate relative response calibration in this range (see Sec. III C). Hence, without resorting to spectral binnings to boost the SNR, which could minimally lower this detection limit, we find the short-wavelength limit of the echelle spectrometer to be $250 \mathrm{~nm}$. In contrast, the upper spectral limit of the full instrument is currently limited to $11.35 \mu \mathrm{m}$ given by the MCT calibration fit (see Fig. 12).

In summary, the free spectral range of the TR spectrometer is $250 \mathrm{~nm}$ to $11.35 \mu \mathrm{m}$ with about $100 \mathrm{~nm}$ spectral overlap between the different spectrometer detectors. This CTR spectrometer spectral range enables resolving bunch durations and periodic temporal bunch modulations on a temporal scale of 0.8 to 38 fs given by the minimum and maximum detection wavelength. Furthermore, periodic spectral features indicating trains of bunches [32] with a characteristic spacing of $L=c T=\lambda_{0}^{2} / \Delta \lambda_{0}$ can also be measured. The smallest periodic spacings are given by broad peaks at the detected wavelength $L=\lambda_{0}$ at $\Delta \lambda_{0}=\lambda_{0}$, while the largest detectible spacings depend on the minimal detector resolution at a given wavelength,

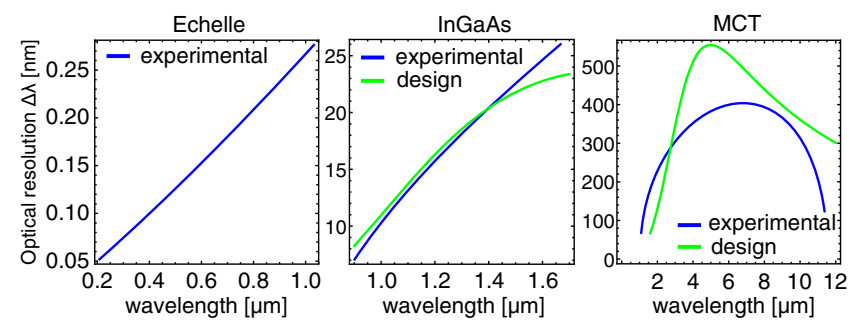

FIG. 13. Features the absolute instrument spectral resolution $\Delta \lambda$, individually obtained for each spectrometer arm. Since the theoretical angular dispersion for the commercial echelle spectrometer is not available, only the experimental curve is presented here.
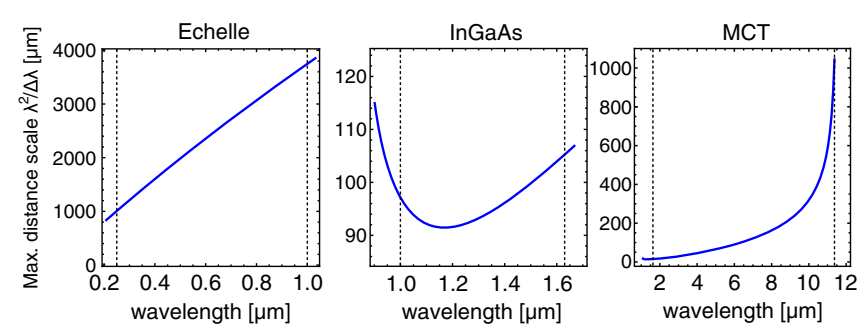

FIG. 14. Shows the maximum bunch spacings $L=c T=$ $\lambda_{0}^{2} / \Delta \lambda_{0}$ from trains of electron bunches [32] detectible by the spectrometer. At any given wavelength, the maximum resolvable distance scale $L$ between two bunches of a periodic bunch signal is defined by the instrument relative resolving power $\lambda / \Delta \lambda$, corresponding to the absolute instrument resolution data of Fig. 13. Each detector's spectrum is delimited by grey lines to indicate the later composite spectral range without overlapping wavelengths.

see Fig. 14. Thus the MIR-arm is sensitive to bunch spacings from 1.6 up to $107 \mu \mathrm{m}$, the NIR-arm from 1.0 up to $104 \mu \mathrm{m}$, and the UV/VIS-arm from $250 \mathrm{~nm}$ to $3.7 \mathrm{~mm}$. Note in particular, if one can measure a signal from a sufficiently coherent TR source in the UV/VIS, that spectrometer arm alone provides more information on bunch train spacings than the NIR and MIR ranges.

\section{Relative response calibration}

This first yield calibration is performed by measuring the relative spectral response of the instrument $T_{\mathrm{LS}}(\lambda)$ in units of [counts or V] to calibrated continuous LS with $S_{\mathrm{LS}}(\lambda)$ denoting the spectral emission characteristic of the LS in [arbitrary energy] units. It reflects the wavelength dependent response of the detectors convoluted with the transmission efficiency of the spectrometer arms. This method enables to determine the relative efficiency of the spectrometer over a broad spectral range at once. Due to diffraction, the incoherent emission of the available LSs cannot be precisely collimated and transported through the spectrometer optics, which leads to a large uncertainty in the absolute yield of the LSs. Therefore, this relative calibration is normalized to unity with respect to the wavelength at which the absolute calibration will be anchored later, see Sec. III D.

For the MIR and NIR ranges, a calibrated BB source [61] is used. Its emission spectrum in the range from 0.5 up to $99 \mu \mathrm{m}$ comes close to an ideal BB spectrum (specified $>99 \%$ emissivity) described by the Planck equation. In the UV and NIR ranges a QTH lamp is used and complemented by the BB radiator and a Deuterium lamp. Here, only the calibration result from QTH is presented.

The relative calibration can thus be expressed by

$T_{\text {Rel }}^{s, p}(\lambda)=\frac{T_{\mathrm{LS}}^{s, p}(\lambda)}{S_{\mathrm{LS}}(\lambda)} \times \frac{1}{R_{\text {mirrors }}^{s, p}(\lambda)} \times \frac{1}{T_{\text {polarizer }}(\lambda)} \quad$ and 


$$
T_{\mathrm{Rel}, \mathrm{N}}^{s, p}(\lambda)=T_{\mathrm{Rel}}^{s, p}(\lambda) / T_{\mathrm{Rel}}^{s, p}\left(\lambda_{\mathrm{Cal}}\right),
$$

where $T_{\mathrm{Rel}}^{s, p}$ represents the transmission of the LS through the spectrometer with respect to s- and p-polarization. $S_{\mathrm{LS}}$ denotes the emission spectrum of the LS, which is derived from an independent calibration measurement (for QTH and Deuterium sources) or from fundamental concepts (Planck's law for the BB source). Since alignment optics slightly alter the original LS spectrum, these contributions $R_{\text {mirrors }}^{s, p}$ as well as transmission through the polarizer $T_{\text {polarizer }}$ are explicitly taken into account. $T_{\text {polarizer }}$ was determined with the aforementioned FTIR spectrometer. The spectral reflectivity of aluminum, gold, and silver coated mirrors is taken from specifications.

Finally, the relative calibration curves are normalized to unity with respect to the value of the central pixel $T_{\mathrm{Rel}}^{s, p}\left(\lambda_{\mathrm{Cal}}\right)$ corresponding to the laser line $\lambda_{\mathrm{Cal}}$ applied for the following absolute calibration.

\section{Absolute calibration}

Equation (12) encodes information about the relative response of the spectrometer as a function of the wavelength. A subsequent energy calibration at a laser line defined reference point thus transforms the entire relative calibration into an absolute. This procedure is performed for s- and p-polarization separately.

For the EMCCD and InGaAs detectors, $532 \mathrm{~nm}$ and $1.31 \mu \mathrm{m} \mathrm{CW}$ laser sources are employed, respectively. For signal acquisition, the detector exposure time is internally set to $\tau_{\exp }=50 \mathrm{~ms}$ in order to mitigate the error in $\tau_{\exp }$ originating from the intrinsic detector gating jitter and readout noise. Additionally, at the input of the echelle spectrometer a programmable mechanical shutter is implemented for minimizing further signal accumulation during readout. Furthermore, the intensity of the laser beams is reduced by neutral density (ND) filters such that the entire dynamic range of the detector is covered. The average power $P_{\text {avg }}$ of the $\mathrm{CW}$ laser beam is measured by a calibrated thermal power detector [62] while the ND attenuators are removed from the beam in order to increase the SNR of the measurement. The involved ND filters are separately calibrated for the same laser beam and included in the final energy calculation. For a CW laser, the energy contained within a specific acquisition time $\tau_{\exp }$ is thus calculated by

$$
E_{\text {laser }}[\mathrm{J}]=P_{\mathrm{avg}}[\mathrm{W}] \times T_{\mathrm{ND}} \times \tau_{\exp }[\mathrm{s}],
$$

where $P_{\mathrm{avg}}$ and $T_{\mathrm{ND}}$ denote the laser average power and the attenuation factor, respectively.

The calibration of the MCT detector requires more effort, since the detector time constant of $400 \mathrm{~ns}$ does not allow to perform valid measurements with pulses longer than this time constant. In the MIR range laser sources that can provide pulses below $400 \mathrm{~ns}$ are limited. One rather complex option is to use accelerator-based FEL LSs [63] as, e.g., provided by HZDR's LS ELBE. Here, we apply an alternative transportable method by adapting a $\mathrm{CO}_{2}$ laser with an acousto-optical modulator (AOM). The AOM enables the generation of short pulses down to $100 \mathrm{~ns}$ with $\mathrm{kHz}$ repetition rate.

The $\mathrm{CO}_{2}$ calibration setup is depicted in Fig. 15. A temperature stabilized quasi-CW $\mathrm{CO}_{2}$ laser with $1 \mathrm{~W}$ output [64] is utilized. The AOM system [65] uses germanium (Ge) for the IR optical interaction material with a lithium niobate transducer. The $\mathrm{CO}_{2}$ laser polarization axis is adjusted to the Bragg plane of the Ge-crystal (here p-polarization) using a holographic wire-grid linear polarizer (WGP1) [66]. The AOM can be externally triggered at $f=1 \mathrm{kHz}$ with an output pulse width of $200 \mathrm{~ns}$ (FWHM).

The p-polarized output pulse train from the AOM setup is thereafter polarized to $45^{\circ}$ by WGP 2 . WGP 3 then defines the final output polarization of the AOM assembly for the MCT detector calibration. The average power $P_{\text {avg }}$ of each polarization is measured using a calibrated thermal power detector [62]. The MCT readout electronic is synchronized to this laser pulse train.

In order to improve the SNR during the power measurement, the laser pulse width is increased by a factor of 1000 to $\tau_{\text {Laser }}=200 \mu$ s. The pulse energy of laser pulse is calculated by $E[\mathrm{~J}]=P_{\mathrm{avg}}[\mathrm{W}] \times f^{-1}[\mathrm{~s}]$ and the pulse energy during the MCT calibration measurement obeys

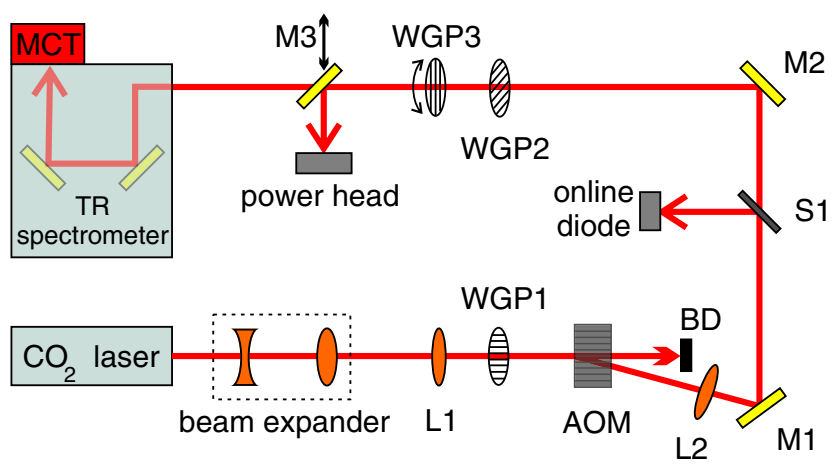

FIG. 15. Schemetic of the $\mathrm{CO}_{2}$ calibration setup: The beam expander consists of a defocusing and a focusing $\mathrm{ZnSe}$ lens, enlarges the beam diameter from $\sim 1$ to $\sim 10 \mathrm{~mm}$. The beam is focused on the AOM crystal by L1 and is polarized by WGP1. The deflected pulsed beam under $161 \mathrm{mrad}$ deflection angle is recollimated by L2. Following the mirror M1, a fraction of the beam is reflected by the $1 \mathrm{~mm}$ thick silicon wafer S1 into a fast photodiode to monitor the laser pulse length. Transmission through the S1 passes WGP2 set at $45^{\circ}$ angle and further passes the adjustable WGP3 which is utilized to define the output polarization of the setup. The mirror M3 can be either inserted into the beam for power measurement or removed for spectrometer calibration. 


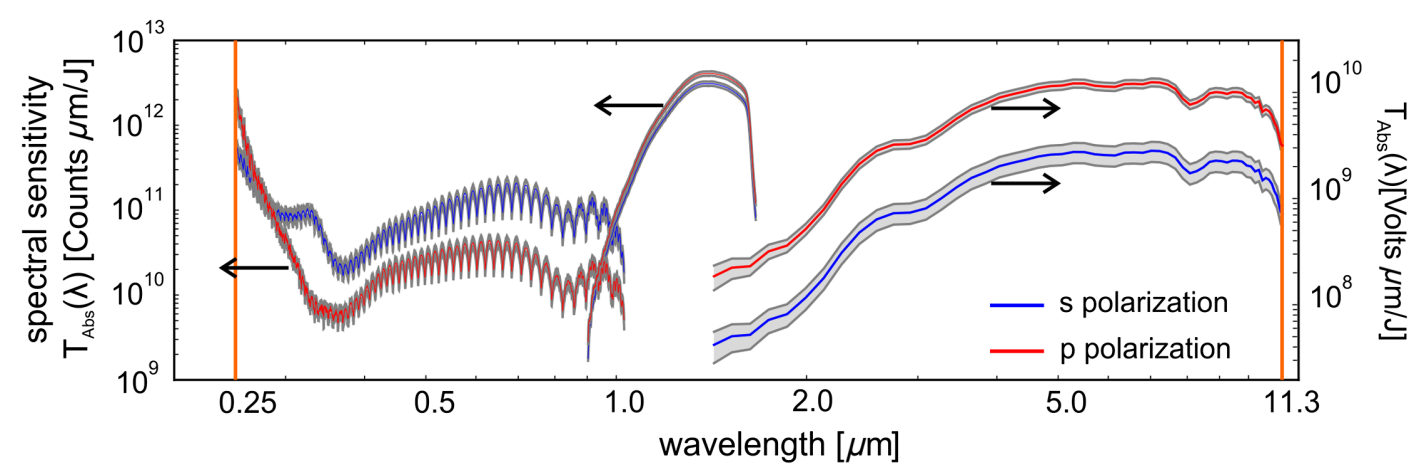

FIG. 16. Overview over the bandwidth-corrected absolute instrument calibration: The three sets of calibration curves represent the spectral range of the three independent detectors. Each set is presented with respect to s- and p-polarization as indicated in blue and red, respectively. The gray area indicates the error contributions from the wavelength, relative, and absolute calibrations. Arrows indicate the associated axes.

$$
E_{\text {laser }}[\mathrm{J}]=\frac{\tau_{\text {exp }}}{\tau_{\text {Laser }}} \times P_{\text {avg }}[\mathrm{W}] \times f^{-1}[\mathrm{~s}] .
$$

The absolute calibration factor is therefore given by

$$
F_{\mathrm{Abs}}^{s, p}=\frac{S_{\text {laser }}}{E_{\text {laser }}} .
$$

$S_{\text {laser }}=\sum_{\#} S_{\text {laser }}(\#)$ denotes the integrated signal from the calibration laser measured over the entire region of interest on the detector and $E_{\text {laser }}$ is given by the Eqs. (13) or (14). The unit of $F_{\mathrm{Abs}}^{s, p}$ depends on the detector [counts $\mathrm{J}^{-1}$ ] or $\left[\mathrm{V} \mathrm{J}^{-1}\right]$.

Finally, a normalization of the absolute calibration curves by the spectral bandwidth $\mathrm{BW}(\lambda)=\Delta \lambda$ covered by the individual detector pixels eliminates the nonlinearity by nonequidistant wavelength sampling of the detectors and thus provides a consistent calibration over the entire spectrum. The bandwidth normalized absolute calibration is therefore given by

$$
\begin{gathered}
T_{\mathrm{Abs}}^{s, p}(\lambda)=T_{\mathrm{Rel, \textrm {N }}}^{s, p}(\lambda) \times F_{\mathrm{Abs}}^{s, p} \text { and } \\
T_{\mathrm{Abs}, \mathrm{BW}}^{s, p}(\lambda)=T_{\mathrm{Rel}, \mathrm{N}}^{s, p}(\lambda) \times F_{\mathrm{Abs}}^{s, p} \times \mathrm{BW}(\lambda),
\end{gathered}
$$

where the quantity $\mathrm{BW}(\lambda)$ indicates the discrete spectral bandwidth of the detector array for each pixel and is derived from the wavelength calibration curve of the corresponding detector (see inset on Fig. 11). The absolute calibration curve $F_{\mathrm{Abs}}^{s, p}$ from Eq. (17) is given in units of counts or voltage $\times \mu \mathrm{m} / \mathrm{J}$ and is summarized in Fig. 16 .

The error band (depicted in gray) reflects one standard deviation and contains all error sources as follows: (i) The relative error from signal noise of each detector $\sigma_{S_{n}}$ is considered as a function of wavelength and calculated by

$$
\sigma_{S_{n}}(\lambda)=\sigma_{\text {Bgd }}(\lambda) / S_{n}(\lambda) .
$$

It depends on the signal level and the noise from the environment such as temperature, readout noise but also electromagnetic pulse events during the acquisition. (ii) The relative error regarding the spectral bandwidth $\mathrm{BW}(\lambda)$ of each detector element is obtained from the standard error estimated from the corresponding wavelength fit function and is calculated by

$$
\sigma_{\Delta \lambda}=\Delta \lambda / \bar{\lambda}
$$

(iii) The error from the relative response calibration $\sigma_{\mathrm{Rel}}$ is estimated according to the absolute calibration of the LS. This error is negligible $(\approx 1 \%)$ for the BB source and lies between $8.2 \%$ and $9.4 \%$ for the QTH lamp in the spectral range of echelle spectrometer. (iv) The largest error contributions originate from the uncertainties in the absolute calibration. The error directly related to the power head calibration is $3 \%$, valid for all utilized calibration lasers. In addition, the individual errors in the power measurements are for the echelle spectrometer 7\%, for the InGaAs 3\%, and for the MCT heads on average $12 \%$.

Note that above calibration procedure identifies the combined responsitivity curve from slit to detected signal. Particularly, this also includes responsitivity variations of individual detector elements. Such procedure implicitly relies on the detectors response to be linear, which was tested and confirmed in the photometric calibration measurements.

For the measurements presented in Sec. IV, appropriate ND filters are added to the NIR and MIR arms of the TR spectrometer for exploiting the full dynamic range of the detectors. Additionally in the MIR arm, KRS-5 holographic WGPs [67] are utilized pairwise, as a cross-polarizer, in order to precisely adjust the throughput to conform to the dynamic range of the InGaAs and MCT detector-resulting in two and four orders of magnitudes signal reduction in the NIR and MIR arms, respectively. A dedicated calibration run for TR spectrometer is performed, in which the aforementioned ND filters are employed. Hence, the absolute calibration given in Eq. (17) can be extended to 


$$
\begin{aligned}
T_{\text {Abs,BW }}^{s, p}(\lambda)= & T_{\text {Rel, } \mathrm{N}}^{s, p}(\lambda) \times T_{\text {polarizer }}^{s, p}(\lambda) \times T_{\mathrm{ND} \text { filter }}^{s, p}(\lambda) \\
& \times T_{\text {beamline }}^{s, p}(\lambda) \times F_{\text {Abs }}^{s, p} \times \mathrm{BW}(\lambda),
\end{aligned}
$$

where $T_{\text {polarizer }}, T_{\mathrm{ND} \text { filter }}$, and $T_{\text {beamline }}$ denote the transmission through the cross polarizers, transmission through the ND filters, and CTR beam line efficiency, respectively. Note, that due to the radial polarization of the TR emission the beam entering into the spectrometer can be assumed to be "unpolarized". In practice, the polarization dependent calibration curves [Eq. (17)] are averaged over s- and p-polarization in order to obtain the unpolarized calibration values [Eq. (20)]. This approximation is valid only if the characteristic diameter of the TR single-electron pointspread-function at a given wavelength $\simeq \sqrt{2} \lambda[11,68,69]$ is significantly smaller than all delimiting (slit) apertures in the spectrometer, p.ex. smaller than the $50 \mu \mathrm{m}$ aperture for the echelle spectrometer.

\section{E. Detection threshold}

For the presented instrument operation over a highdynamic range is inherently coupled to high sensitivity. The latter is characterized via the noise-equivalent power defined as the power $\phi$ radiated onto the detector which yields a SNR of unity [70]. In order to provide an estimate of the minimum energy that can be detected, the NEE is introduced representing the rms noise level and is given in units of $\mathrm{J} / \mu \mathrm{m}$ as

$$
\mathrm{NEE}=\frac{\sigma_{\phi}}{T_{\mathrm{Abs}, \mathrm{BW}}^{s, p}(\lambda)}
$$

Here $\sigma_{\phi}$ denotes the standard deviation of the statistical shot-to-shot variations in background acquisitions and $T_{\text {Abs,BW }}^{s, p}$ the absolute BW-normalized response of the spectrometer according to Eq. (17). In the Fig. 17, the NEE is depicted for the entire spectral range of the TR spectrometer and with respect to the polarization of the incident beam.

The lowest detection threshold is achieved for the MCT and InGaAs detectors at $2 \mathrm{pJ} / \mu \mathrm{m}$. The echelle spectrometer shows a detection threshold of about $100 \mathrm{pJ} / \mu \mathrm{m}$. The latter

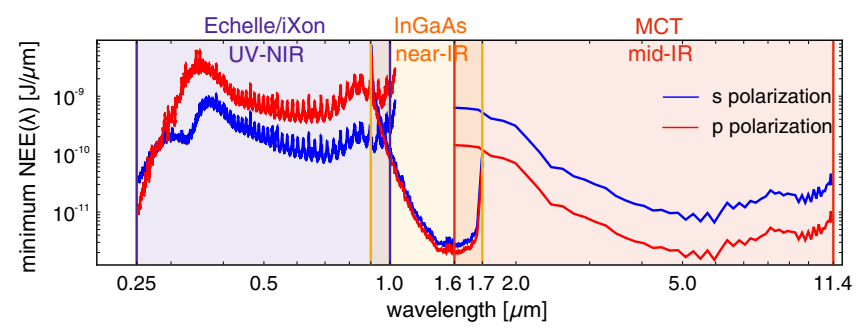

FIG. 17. Noise-equivalent energy (NEE) of the TR spectrometer: The NEE is estimated for the three spectral ranges and appropriate detection systems with respect to s- and p-polarization at maximum achievable resolution. could be significantly improved by increasing its EM gain, which here is set at $30 \times$ amplification. However, an increase in the EM gain reduces the dynamic range of the detector.

The sensitivity of each spectrometer arm can be individually adapted to the dynamic range matching an application by means of attenuators or polarizers in either spectrometer arm. The NIR spectrum reveals less polarization dependency than the echelle and MIR spectra. This is due the nearly polarization independent transmission and reflection properties of optical elements of the spectrometer. In particular at $\lambda=1.31 \mu \mathrm{m}$ this deviation is about $25 \%$ from the mean.

According to the spectral radiation energy emitted by a single electron $W_{\mathrm{TR}} / d \omega \simeq(2 / \pi) r_{e} m_{e} c \log (\gamma)$ [28], while assuming $300 \mathrm{MeV}$ electrons, one can estimate the minimum number of electrons $N_{e}$ required by a spatially compact bunch to generate a fully coherent CTR signal $\left(Q_{\omega, \mathrm{thr}} / e\right)^{2}=N_{e, \min }^{2}=\int N E E_{\omega} /\left(W_{\mathrm{TR}} / d \omega\right) d \omega . \quad$ Taking into account the $2 \mathrm{pJ} / \mu \mathrm{m}$ minimum NEE at $5.5 \mu \mathrm{m}$ in p-polarization, one arrives at a detection threshold of $Q_{\omega, \text { thr }} \sim 23 \mathrm{fC}$. Due to its high-frequency resolution the NEE in the UV or VIS arm is significantly higher compared to the MIR. However, post-analysis spectral binning at $\sim 10 \mathrm{~nm}$ resolution leads to similar detection thresholds. Taking into account spectral variations in NEE of up to two orders of magnitudes in each spectrometer arm, CTR thus becomes independently detectable at each spectrometer wavelength for electron bunch charges as low as the pC-scale.

\section{EXPERIMENTAL RESULTS}

In order to benchmark the spectrometer under realistic experimental conditions, it was employed in a series of LWFA experiments. Following acceleration ultrashort electron bunches in the pulse duration range of $10 \mathrm{fs}$ pass through a thin metallic foil and generate TR in forward direction. The emitted TR spectrum, characteristic for the temporal profile of the electron bunch, is typically broadband and covering a large dynamic range. Its full measurement enables the deconvolution of the temporal structure of the electron bunch in an independent postprocessing step [18].

The experiments were performed at HZDR using the 100 TW DRACO laser system [71]. It delivers 30 fs laser pulses with energies up to $3.5 \mathrm{~J}$ on target. A $3 \mathrm{~mm}$ de Laval nozzle [72] is used providing a supersonic jet composed of a $98.5 \%-\mathrm{He}+1.5 \%-\mathrm{N}_{2}$ gas mixture. When fully ionized, an electron density of $n_{\mathrm{e}}=3.4 \times 10^{18} \mathrm{~cm}^{-3}$ is generated which corresponds to a linear plasma wavelength of $\lambda_{\mathrm{p}}=17.1 \mu \mathrm{m}$. For electron injection and acceleration the self-truncated ionization injection (STII) scheme in the bubble-regime is exploited [30,73]. Typically, electron bunches are accelerated up to $400 \mathrm{MeV}$ with a peak charge 
of several hundred $\mathrm{pC}(\mathrm{FWHM})$ at relative energy spread of about $10 \%$.

For the bunch duration measurement, a $5 \mu \mathrm{m}$ steel foil located $26 \mathrm{~mm}$ downstream of the nozzle exit is used as the TR source. Before, the laser is blocked by a double 40-Aluminum foil tape drive, whereas the electrons pass through to the subsequent TR target. A 3-in. aluminumcoated SM (F/31) recollimates the forward TR. It is followed by five 2 -in. aluminum-coated folding mirrors to transport the TR beam into the TR-spectrometer. The first off-axis-parabolic mirror (AL-OAP1) (see Fig. 2) images the TR source onto the $200 \mu \mathrm{m}$ entrance slit of the instrument. The electron beam divergence is measured to about $5 \mathrm{mrad}$ (rms) leading to a transverse electron beam size on the TR screen of less than $500 \mu \mathrm{m}$. Thus, the F/31 SM leads to an image size of $60 \mu \mathrm{m}$ in diameter fitting into the entrance slit and preventing clipping of the TR beam for many different LWFA beams. Note that this condition only guarantees that the TR beam is fully captured by the far-IR arm. For the UV/VIS and near-IR arm, subsequent slit sizes of 50 and $100 \mu \mathrm{m}$ are smaller, respectively. Thus, beam size and TR beam pointing can lead to clipping and consequent signal reduction. However, we monitor the entrance slit for each shot by inserting a beam sampler in front of the echelle spectrometer, which reflects $1.0 \%$ of the visible TR into the imaging optics.

The high charge accelerated in the STII scheme leads to CTR emission of intensities up to tens of $\mu \mathrm{J} / \mu \mathrm{m}$ especially in the infrared range. Figure 18 shows a typical set of raw and calibrated data acquired by the three spectrometer arms. Notably, the raw spectrum from the echelle spectrometer shows an extremely modulated signal as expected from varying grating efficiency. The lack of this signature in the corrected spectrum illustrates the high quality of the calibration. The corrected MCT spectrum shows a smoothly rising signal towards the long wavelength range. The latter encodes information on the overall electron bunch duration which is only accessible with a proper absolute calibration. The NIR spectrum provides the connection between the three arms. The sharp cutoff in the raw InGaAs spectrum above $1.58 \mu \mathrm{m}$ is due to the strong decrease in its response which is partially corrected by the calibration. Nonetheless, the overlapping regions between the arms coincide reasonably well, in particular when regarding the spectral evolution. The ratio between the measured intensities by the echelle spectrometer and InGaAs detector at $\lambda=1.0 \mu \mathrm{m}$ is $W_{\text {InGaAs }} / W_{\text {Echelle }}=4.6$. On the other hand, the ratio between the MCT and InGaAs at $\lambda=1.63 \mu \mathrm{m}$ is $W_{\mathrm{MCT}} / W_{\text {InGaAs }}=3.8$.

For merging the partial spectra, when full beam capture can be assumed for all detectors, the NIR spectrum serves as the reference with respect to its absolute intensities. Benefitting from the imaging geometry of the beam transport into the detector, the NIR is less sensitive to beam pointing compared to the echelle spectrum.
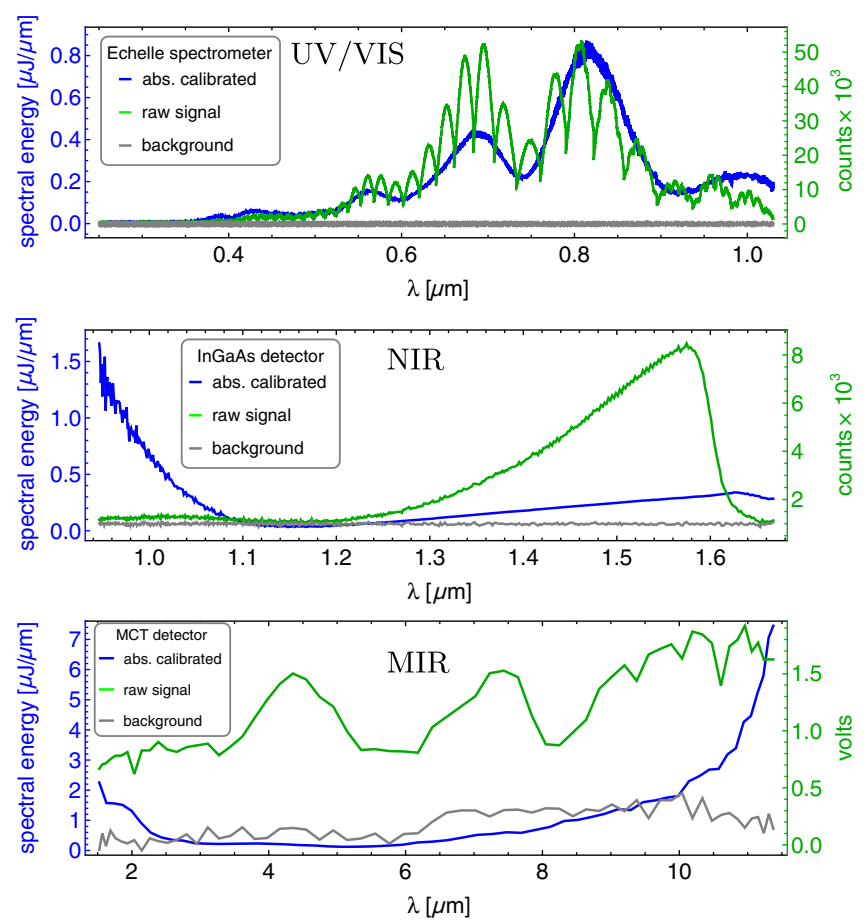

FIG. 18. Typical spectral measurement of coherent to incoherent TR radiation: The absolutely calibrated and bandwidth normalized partial spectra, depicted in blue, are determined using Eq. (20). The green curves show the raw signal acquired by the associated detectors. The gray curves, associated also with the right axis, show the background signal acquired by removing the TR screen and SM collimator from the beam axis.

Additionally, its calibration is more precise than the MIR spectrum due to its nearly polarization independent spectral range. Thus, the NIR and echelle spectra are rescaled with respect to their response at the range boundaries at $\lambda_{\mathrm{MCT} \text {,min }}=1.6 \mu \mathrm{m}$ and $\lambda_{\text {Echelle, } \max }=1.0 \mu \mathrm{m}$. In the contrasting case of partial (CTR) beam capture in the NIR and UV-VIS range, but still full beam capture in the MIR, it is the MIR spectrum, which is then used as an auxiliary photometric reference. Figure 19 summarizes the result of such a re-scaled spectrum with the connecting wavelength values being marked by vertical dashed lines. When comparing the responses in the overlap regions, the slopes of the UV-VIS and MIR spectral curves agree well with the NIR spectral curve progression $\leq 1.0 \mu \mathrm{m}$ and at $1.6 \mu \mathrm{m}$, respectively. It is worth noting that the pronounced InGaAs deviations below $1.0 \mu \mathrm{m}$ compared to the echelle spectrum, cannot be explained by calibration uncertainties alone. Particularly the low spectral sensitivity due to the GaAs beam splitter cut-off when combined with a low SNR makes this spectral range of the InGaAs arm susceptible to minimal drifts in background or fine-alignment. Thus, we exclude the InGaAs detector data below $1.0 \mu \mathrm{m}$.

Figure 20 presents the extended TR spectrum. The three spectral ranges (Fig. 19) are combined and subsequently transformed to the frequency domain. The error band 

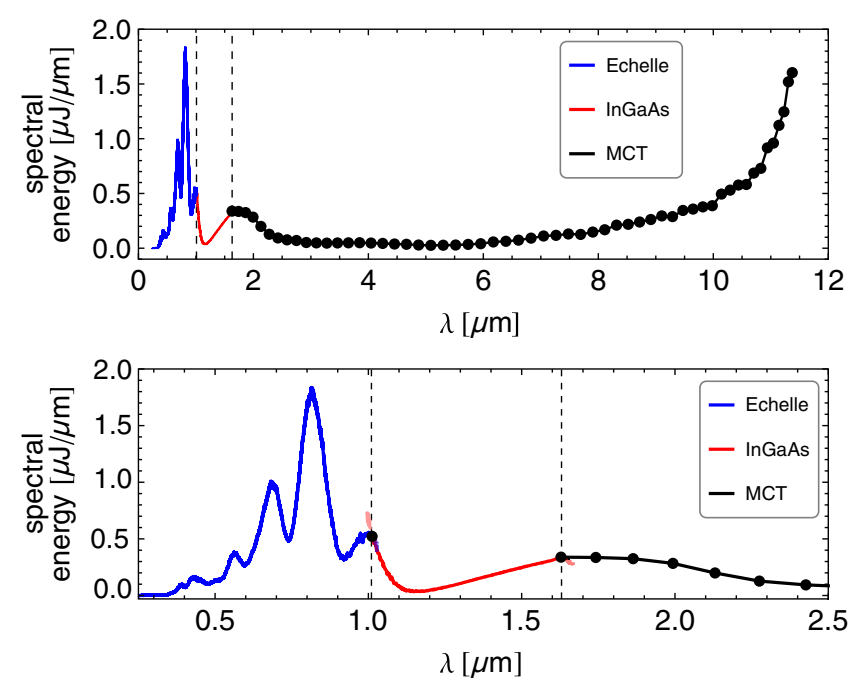

FIG. 19. (a) Combined measured TR spectrum: The Echelle and MCT spectra are rescaled in their intensities according to overlap wavelengths to the InGaAs spectrum. The overlap wavelengths are $\lambda=1.0 \mu \mathrm{m}$ and $1.63 \mu \mathrm{m}$ for Echelle and MCT spectra, respectively. (b) depicts a zoomed-in spectrum with InGaAs (pink) and Echelle data (transparent blue) beyond their designated spectral domains in regions of overlapping wavelengths.

(depicted in gray) indicates a standard deviation of the mean. It is separately shown on the bottom of Fig. 20 as a function of frequency. In the error analysis, the contribution of the uncertainties of the wavelength calibration and relative calibration from each partial spectra as well as the contribution of the absolute calibration uncertainties from the NIR spectrum are taken into account.

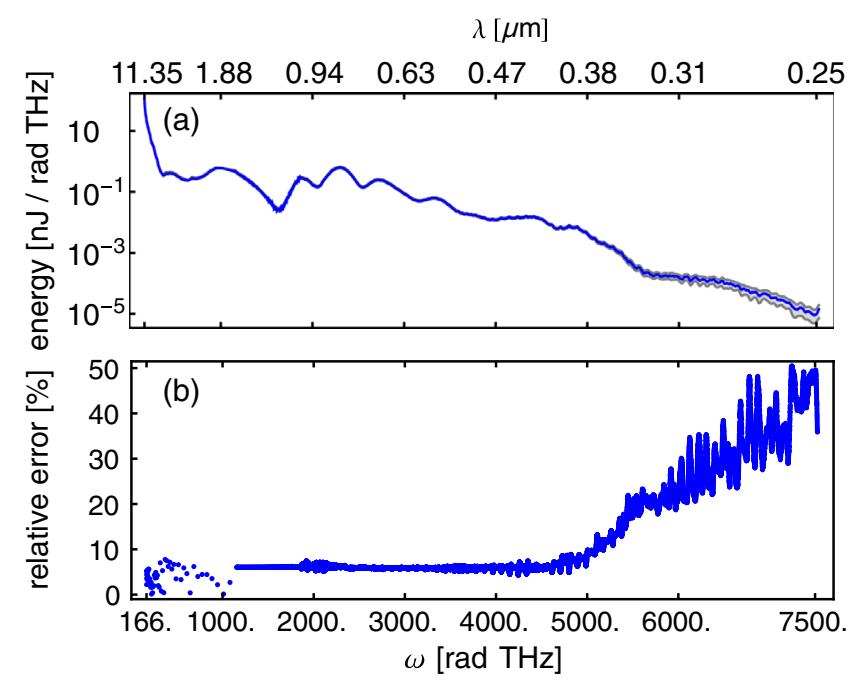

FIG. 20. (a) shows the TR spectrum of Fig. 19 in the frequency domain, combined from all three spectrometer arms. (b) depicts the corresponding relative error, dominating the UV-part of the instrument.

\section{DATA ANALYSIS}

The data analysis and reconstruction procedure closely follows [18], which is based on a variant of the iterative Hybrid-Input-Output method [74,75]. This includes adaptive support and positive-definite charge density constraints in the real-space domain, i.e., the bunch profile over time. The spectral domain is constrained by the modulus of the form factor $|F(\omega)|$, as determined by experiment. Additionally, the zero-frequency normalization and oddphase constraint enforce full coherence at $\omega=0$ and real valued form factor $F(t)$, respectively.

In addition to the measured CTR spectrum, we take into account the simultaneously acquired electron spectrum for charge, energy and divergence, as well as the acceptance angle $\theta_{\max }$ of the TR collection optics. The measured data is used to compute the hypothetical, fully coherent TR spectrum, which when compared to the actually measured CTR spectrum yields the modulus of the CTR form factor distribution $|F(\omega)|$. The latter is required as input for the subsequent phase-reconstruction procedure [18] to obtain the complex-valued $F(\omega)$ and thus $F(t)$, as well as the beam current profile $I(t)$.

Starting from random phase for a complex-valued form factor $F(\omega)$, based on one given experimental spectrum, the reconstruction algorithm is performed in total 150 times, yielding a range of reconstruction candidates. To account for the predominantly systematic measurement uncertainties, 50 of the 150 reconstructions are performed on the measured data, the other $2 \times 50$ reconstructions are applied on variations to this initial data set in which the relative spectral error characteristics from calibration (Fig. 19) are added to and subtracted from the data set, respectively. Before reconstruction candidates can be compared, these are classified and normalized for possible temporal-shift and time-inversion ambiguities inherent to the reconstruction procedure. By maximizing the correlation to some reference reconstruction the temporal shifts of the candidate solutions, as well as the possible time inversion, are made consistent across candidate solutions. As initial reference we use the Kramers-Kronig (KK) reconstruction procedure. Although the KK procedure features considerable weaknesses [18,27,29] in reconstructing smooth, temporally extended bunch features, it is fast and robust in detecting localized sub-structures in complex beams, which for structured beams simplifies correlation and thus temporal alignment of all candidate solutions. Based on these correlated reconstruction candidates, a mean longitudinal density profile with corresponding standard deviations is generated.

The resulting electron bunch reconstruction, Fig. 21, yields a pulse duration of $\tau_{e}=(9.78 \pm 0.77)$ fs and a peak current of $I_{e}=(9.40 \pm 0.66) \mathrm{kA}$ with relative standard deviations of $7.87 \%$ and $7.02 \%$, respectively. On a short time scale, bunch sub-structures with a typical periodicity of $\sim 3.0 \mathrm{fs}$ occur. The reconstructed fs-scale modulation of 


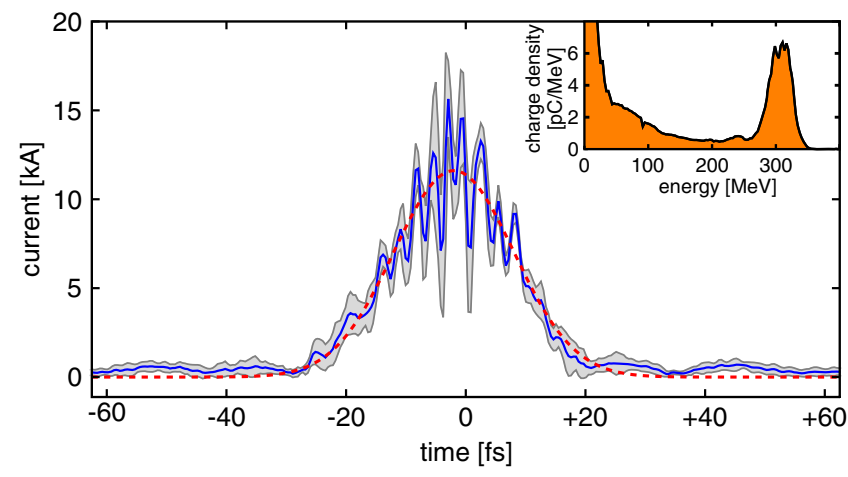

FIG. 21. Features a reconstructed ultrashort Laser-wakefield accelerated electron bunch based on the CTR spectrum, see Fig. 19, the electron spectrum, bunch charge and bunch divergence acquired at single-shot. The electron bunch features $(9.78 \pm 0.77)$ fs rms-bunch duration, $(9.40 \pm 0.66) \mathrm{kA}$ peak current and sub-structure with $\sim 3.0$ fs characteristic periodicity.

the ultrashort electron bunch further highlights how the instrument makes use of its full spectral range to simultaneously measure electron bunch structures at disparate time scales.

The errors on bunch duration and current, include the errors from spectral measurement, calibration (see Fig. 21) and the reconstruction uncertainty by remaining ambiguities in the data.

The systematic, normalization error by uncertainties in the power measurements dominates the spectral measurement. Thus the reconstruction procedure was repeated for each the maximum and the minimum spectral intensities consistent with the error interval characteristics in Fig. 20. Particularly this varies the degree of coherence of the TR emitted by the electron bunch, which directly impacts possible bunch length, as well as sub-bunch features.

For the electron bunch duration, the normalization errors arising from absolute spectral power calibration (5.2\%) are on a similar magnitude than the reconstruction uncertainties $(5.9 \%)$. By contrast, for the electron bunch peak current as acquired by the Gaussian fit to the reconstructed charge distribution, the normalization errors have a much reduced impact $(1.4 \%)$ compared to the reconstruction uncertainty $(6.9 \%)$, but dominate the uncertainty on the quantitative amplitude of the oscillatory sub-structures.

A comparison with Fig. 20(b) for length scales $>400 \mathrm{~nm}$ shows the relative measurement errors of the reconstructed bunch duration quantities to be on a similar magnitude. As normalization errors in measured CTR spectral energy remain a dominant source of systematic uncertainty, it thus can be expected that efforts in minimizing such errors will directly benefit more accurate bunch duration measurements, even if bunch small-scale sub-structures are present.

\section{CONCLUSIONS}

Summarizing, we presented the design, setup, full characterization, and first test of a spectrometer, which is suitable for measuring multioctave TR spectra of ultrashort electron beams in a single shot. We provide the detailed wavelength, relative response, and absolute photometric calibration procedure for each spectrometer arm and with respect to s- and p-polarization. This is done by using a compact assembly of calibration LSs in a tabletop setup below the instrument.

Each of the spectrometer arms can be independently operated allowing for more flexibility in measuring broadband spectra with large variations in intensity by tailoring filter or detector configurations to typical signals. The modular setup facilitates a straightforward extension of its spectral range beyond $20 \mu \mathrm{m}$ (far-IR), e.g., by employing a further similar prism spectrometer to the existing MIR arm. This would be advantageous especially for measuring electron bunch durations significantly beyond 20 fs electron durations. Previous measurements using electro-optic methods [12] have shown that particularly pronounced low-energy electron contributions from parasitic electron injections can cause trailing bunch tails of several hundredfs duration. Although the present CTR spectrometer cannot detect such low-energy bunch tails, acquired results are consistent in characterizing the bunch profiles at high energies, because CTR signals from low-energy electrons $<30 \mathrm{MeV}$ are strongly suppressed due to the $16 \mathrm{mrad}$ acceptance half-angle and the $1 / \gamma$-angular-dependency of TR.

The regions of spectral overlap between the three arms enable cross-checking the stability of calibration and alerting to potential changes in alignment. Due to its large angle of acceptance the mid-IR arm can act as an auxiliary photometric reference for the other arms in case their signals are partially clipped in secondary apertures. For monitoring beam transmission through the spectrometer entrance slit, additionally we installed a camera picking up and imaging $1 \%$ of the signal before the echelle spectrometer, hence enabling diagnostics on eventual beam pointing issues.

The resulting spectrometer enables quantitative studies of highly modulated spectra over a broad spectral range (5.5 octaves, $250 \mathrm{~nm}$ to $11.35 \mu \mathrm{m}$ ) at single shot. This is demonstrated by measuring a broadband TR spectrum CTR spectra from ultrashort laser-wakefield accelerated electron bunches, featuring a dynamic range of more than seven orders of magnitude. Such measurements provide the basis for longitudinal density profile reconstructions [18], allowing to simultaneously resolve features from both the sub-fs to few-10 fs scale. 


\section{ACKNOWLEDGMENTS}

This work was partially funded by Enhanced European Coordination for Accelerator Research \& Development (EUCARD2) under Grand Agreement No. 312453. This project is fully supported by the Helmholtz association under program Matter and Technology, topic Accelerator Research and Development. We wish to thank M. Sobiella, A. Winter, M. Klopf, S. Grams, C. Eisenmann, and M. Werner for their technical support.

[1] A. H. Lumpkin, R. Dejus, J. W. Lewellen, W. Berg, S. Biedron, M. Borland, Y. C. Chae, M. Erdmann, Z. Huang, K.-J. Kim, Y. Li, S. V. Milton, E. Moog, D. W. Rule, V. Sajaev, and B.X. Yang, Evidence for Microbunching "Sidebands" in a Saturated Free-Electron Laser Using Coherent Optical Transition Radiation, Phys. Rev. Lett. 88, 234801 (2002).

[2] P. Chen, J. M. Dawson, R. W. Huff, and T. Katsouleas, Acceleration of Electrons by the Interaction of a Bunched Electron Beam with a Plasma, Phys. Rev. Lett. 54, 693 (1985).

[3] C. Joshi and T. Katsouleas, Plasma accelerators at the energy frontier and on tabletops, Phys. Today 56, No. 6, 47 (2003).

[4] M. Litos et al., High-efficiency acceleration of an electron beam in a plasma wakefield accelerator, Nature (London) 515, 92 (2014).

[5] T. Kurz et al., Demonstration of a compact plasma accelerator powered by laser-accelerated electron beams, Nat. Commun. 12, 2895 (2021).

[6] J.C. Williamson, J. Cao, H. Ihee, H. Frey, and A.H. Zewail, Clocking transient chemical changes by ultrafast electron diffraction, Nature (London) 386, 159 (1997).

[7] Y. Jiang, L. C. Liu, A. Sarracini, K. M. Krawczyk, J. S. Wentzell, C. Lu, R. L. Field, S. F. Matar, W. Gawelda, H. M. Müller-Werkmeister, and R. J.D. Miller, Direct observation of nuclear reorganization driven by ultrafast spin transitions, Nat. Commun. 11, 1530 (2020).

[8] T. Tajima and J. M. Dawson, Laser Electron Accelerator, Phys. Rev. Lett. 43, 267 (1979).

[9] E. Esarey, C. B. Schroeder, and W. P. Leemans, Physics of laser-driven plasma-based electron accelerators, Rev. Mod. Phys. 81, 1229 (2009).

[10] S. Corde, K. Ta Phuoc, G. Lambert, R. Fitour, V. Malka, A. Rousse, A. Beck, and E. Lefebvre, Femtosecond x rays from laser-plasma accelerators, Rev. Mod. Phys. 85, 1 (2013).

[11] M. C. Downer, R. Zgadzaj, A. Debus, U. Schramm, and M. C. Kaluza, Diagnostics for plasma-based electron accelerators, Rev. Mod. Phys. 90, 035002 (2018).

[12] A. D. Debus et al., Electron Bunch Length Measurements from Laser-Accelerated Electrons Using Single-Shot THz Time-Domain Interferometry, Phys. Rev. Lett. 104, 084802 (2010).

[13] O. Lundh, J. Lim, C. Rechatin, L. Ammoura, A. BenIsmaïl, X. Davoine, G. Gallot, J.-P. Goddet, E. Lefebvre, V. Malka, and J. Faure, Few femtosecond, few kiloampere electron bunch produced by a laser-plasma accelerator, Nat. Phys. 7, 219 (2011).

[14] A. Buck, M. Nicolai, K. Schmid, C. M. S. Sears, A. Sävert, J. M. Mikhailova, F. Krausz, M. C. Kaluza, and L. Veisz, Real-time observation of laser-driven electron acceleration, Nat. Phys. 7, 543 (2011).

[15] M. Heigoldt, A. Popp, K. Khrennikov, J. Wenz, S. W. Chou, S. Karsch, S. I. Bajlekov, S. M. Hooker, and B. Schmidt, Temporal evolution of longitudinal bunch profile in a laser wakefield accelerator, Phys. Rev. ST Accel. Beams 18, 121302 (2015).

[16] M. J. H. Luttikhof, A. G. Khachatryan, F. A. van Goor, and K.-J. Boller, Generating Ultrarelativistic Attosecond Electron Bunches with Laser Wakefield Accelerators, Phys. Rev. Lett. 105, 124801 (2010).

[17] X. L. Xu, C.-H. Pai, C. J. Zhang, F. Li, Y. Wan, Y. P. Wu, J. F. Hua, W. Lu, W. An, P. Yu, C. Joshi, and W. B. Mori, Nanoscale Electron Bunching in Laser-Triggered Ionization Injection in Plasma Accelerators, Phys. Rev. Lett. 117, 034801 (2016).

[18] O. Zarini, A. Kohler, J. Couperus, R. Pausch, T. Kurz, S. Schobel, H. Meibner, M. Bussmann, U. Schramm, A. Irman, and A. Debus, Advanced methods for temporal reconstruction of modulated electron bunches, in Proceedings of 2018 IEEE Advanced Accelerator Concepts Workshop, ACC 2018 (2019), p. 1, 10.1109/AAC.2018 .8659388 .

[19] G. Berden, S. P. Jamison, A. M. MacLeod, W. A. Gillespie, B. Redlich, and A. F. G. van der Meer, Electro-Optic Technique with Improved Time Resolution for Real-Time, Nondestructive, Single-Shot Measurements of Femtosecond Electron Bunch Profiles, Phys. Rev. Lett. 93, 114802 (2004).

[20] G. Berden, W. A. Gillespie, S. P. Jamison, E.-A. Knabbe, A. M. MacLeod, A. F. G. van der Meer, P. J. Phillips, H. Schlarb, B. Schmidt, P. Schmüser, and B. Steffen, Benchmarking of Electro-Optic Monitors for Femtosecond Electron Bunches, Phys. Rev. Lett. 99, 164801 (2007).

[21] FESCA-100 Femtosecond streak camera C11853-01 (Hamamatsu).

[22] J. Maxson, D. Cesar, G. Calmasini, A. Ody, P. Musumeci, and D. Alesini, Direct Measurement of sub-10 fs Relativistic Electron Beams with Ultralow Emittance, Phys. Rev. Lett. 118, 154802 (2017).

[23] B. Marchetti, X-band TDS project, in Proceedings of IPAC 2017, MOPAB044 (JACoW, Geneva, 2017), 10.18429/ JACoW-IPAC2017-MOPAB044.

[24] A. J. Gonsalves et al., Petawatt Laser Guiding and Electron Beam Acceleration to $8 \mathrm{gev}$ in a Laser-Heated Capillary Discharge Waveguide, Phys. Rev. Lett. 122, 084801 (2019).

[25] H. Harrison, G. Doucas, A. J. Lancaster, I. V. Konoplev, H. Zhang, A. Aryshev, M. Shevelev, N. Terunuma, and J. Urakawa, The design of a non-destructive single-shot longitudinal bunch profile monitor using Smith-Purcell radiation, in Proceedings of International Particle Accelerator Conference (IPAC'17), Copenhagen, Denmark, 2017 (JACoW, Geneva, Switzerland, 2017), pp. 433-436, 10.18429/JACoW-IPAC2017-MOPAB128. 
[26] V. L. Ginzburg and I. M. Frank, Radiation from a uniformly, moving electron passing from one medium to another, Zh. Eksp. Teor. Fiz. 16, 15 (1946).

[27] B. Schmidt, S. Wesch, T. Kövener, C. Behrens, E. Hass, S. Casalbuoni, and P. Schmüser, Longitudinal bunch diagnostics using coherent transition radiation spectroscopy, arXiv:1803.00608.

[28] C. B. Schroeder, E. Esarey, J. van Tilborg, and W. P. Leemans, Theory of coherent transition radiation generated at a plasma-vacuum interface, Phys. Rev. E 69, 016501 (2004).

[29] S. I. Bajlekov, M. Heigoldt, A. Popp, J. Wenz, K. Khrennikov, S. Karsch, and S. M. Hooker, Longitudinal electron bunch profile reconstruction by performing phase retrieval on coherent transition radiation spectra, Phys. Rev. ST Accel. Beams 16, 040701 (2013).

[30] J. P. Couperus, R. Pausch, A. Köhler, O. Zarini, J. M. Krämer, M. Garten, A. Huebl, R. Gebhardt, U. Helbig, S. Bock, K. Zeil, A. Debus, M. Bussmann, U. Schramm, and A. Irman, Demonstration of a beam loaded nanocoulombclass laser wakefield accelerator, Nat. Commun. 8, 487 (2017).

[31] K. Németh, B. Shen, Y. Li, H. Shang, R. Crowell, K. C. Harkay, and J. R. Cary, Laser-Driven Coherent Betatron Oscillation in a Laser-Wakefield Cavity, Phys. Rev. Lett. 100, 095002 (2008).

[32] O. Lundh, C. Rechatin, J. Lim, V. Malka, and J. Faure, Experimental Measurements of Electron-Bunch Trains in a Laser-Plasma Accelerator, Phys. Rev. Lett. 110, 065005 (2013).

[33] J. M. Lerner and R. M. Zucker, Calibration and validation of confocal spectral imaging systems, Cytometry Part A 62A, 8 (2004).

[34] P. Martinsen, B. Jordan, A. McGlone, P. Gaastra, and T. Laurie, Accurate and precise wavelength calibration for wide bandwidth array spectrometers, Appl. Spectrosc. 62, 1008 (2008).

[35] X. Du, C. Li, Z. Xu, and Q. Wang, Accurate wavelength calibration method for flat-field grating spectrometers, Appl. Spectrosc. 65, 1083 (2011).

[36] M. D'orazio and B. Schrader, Calibration of the absolute spectral response of Raman spectrometers, J. Raman Spectrosc. 2, 585 (1974).

[37] W. S. Hurst, S. J. Choquette, and E. S. Etz, Requirements for relative intensity correction of Raman spectra obtained by column-summing charge-coupled device data, Appl. Spectrosc. 61, 694 (2007).

[38] W. Demtroeder, Laser Spectroscopy, 4th ed. (SpringerVerlag, Berlin, 2008), Vol. 1.

[39] T. Wilson, The role of the pinhole in confocal imaging system, in Handbook of Biological Confocal Microscopy, edited by J. B. Pawley (Springer US, Boston, MA, 1995), pp. 167-182.

[40] M. C. Hutley, Diffraction Gratings (Academic Press, New York, 1982).

[41] D. T. F. Marple, Refractive index of ZnSe, ZnTe, and CdTe, J. Appl. Phys. 35, 539 (1964).

[42] D. Meschede, Optics, light and lasers: The practical approach to modern aspects of photonics and laser physics (John Wiley \& Sons, New York, 2017).
[43] G. Ghosh, Sellmeier coefficients and dispersion of thermooptic coefficients for some optical glasses, Appl. Opt. 36, 1540 (1997).

[44] Wolfram Research, Mathematica (Wolfram Inc., Champaign, Illinois, 2009).

[45] N. Hagen and T. S. Tkaczyk, Compound prism design principles, I, Appl. Opt. 50, 4998 (2011).

[46] N. Hagen and T.S. Tkaczyk, Compound prism design principles, II: triplet and Janssen prisms, Appl. Opt. 50, 5012 (2011).

[47] N. Hagen and T.S. Tkaczyk, Compound prism design principles, III: Linear-in-wavenumber and optical coherence tomography prisms, Appl. Opt. 50, 5023 (2011).

[48] SCHOTT glass data sheets, N-SF11 (2014).

[49] D. T. F. Marple, Refractive index of ZnSe, ZnTe, and CdTe, J. Appl. Phys. 35, 539 (1964).

[50] Andor Technology, Belfast, UK, Echelle spectrograph Mechelle (ME5000) (2011).

[51] A. K. Gaigalas, L. Wang, H.-J. He, and P. DeRose, Procedures for wavelength calibration and spectral response correction of CCD array spectrometers, J. Res. Natl. Inst. Stand. Technol. 114, 215 (2009).

[52] N. Bibinov, H. Halfmann, P. Awakowicz, and K. Wiesemann, Relative and absolute intensity calibrations of a modern broadband echelle spectrometer, Meas. Sci. Technol. 18, 1327 (2007).

[53] Andor Technology, Belfast, UK, 14-bit, back illuminated EMCCD iXon3 888 (A-DU888-EC-BVF) (2012).

[54] Andor Technology, Belfast, UK, InGaAs array-detector iDus 490 (A-DU490A-1.7) (2012).

[55] Infrared Systems Development, 64-element HgCdTe detector array (MCT-64-0.2x0.4 with IR-6416 electronics (2009).

[56] A. Rogalski, Infrared Detectors (CRC press, Boca Raton, 2010), ISBN 9781420076721.

[57] H. Budzier and G. Gerlach, Thermal Infrared Sensors: Theory, Optimisation and Practice (John Wiley \& Sons, New York, 2011).

[58] Andor Technology, Belfast, UK, 1 way fiber, single $50 \mu \mathrm{m}$ core, UV/VIS, SMA-SMA, 2m (A-ME-OPT-8004).

[59] Thorlabs, Fiber-Coupled Laser Source, $1310 \mathrm{~nm}, 1.5 \mathrm{~mW}$, PM Fiber, FC/PC (S1FC1310PM).

[60] Ocean Optics, Argon Calibration Source, spectral lines 696-1704 nm (AR-1).

[61] Infrared Systems Development, calibrated, temperaturecontrolled black body source $\left(50-1050{ }^{\circ} \mathrm{C}\right)$ (IR-508/301) (2009).

[62] Gentec, calibrated thermal power detector (XLP12).

[63] F. Gabriel, P. Gippner, E. Grosse, D. Janssen, P. Michel, H. Prade, A. Schamlott, W. Seidel, A. Wolf, and R. Wünsch, The Rossendorf radiation source ELBE and its FEL projects, Nucl. Instrum. Methods Phys. Res., Sect. A 161-163, 1143 (2000).

[64] Soliton Laser und Messtechnik GmbH, $1 \mathrm{~W} \mathrm{CO}_{2}$ Laser (L4).

[65] ELS Elektronik Laser GmbH, Acousto-optic modulator system (N37041-1).

[66] Thorlabs, $\mathrm{BaF}_{2}$ holographic wire-grid linear polarizer (WP25H-B).

[67] Thorlabs, KRS-5 holographic wire-grid linear polarizer (WP50H-K). 
[68] M. Castellano and V. A. Verzilov, Spatial resolution in optical transition radiation beam diagnostics, Phys. Rev. ST Accel. Beams 1, 062801 (1998).

[69] X. Artru, R. Chehab, K. Honkavaara, and A. Variola, Resolution power of optical transition radiation: Theoretical considerations, Nucl. Instrum. Methods Phys. Res., Sect. B 145, 160 (1998).

[70] A. Daniels, Infrared Systems, Detectors, and FPAs, 3rd ed., edited by J. E. Grievenkamp, Field Guide (SPIE, 2018), ISBN 978-1510618640.

[71] U. Schramm et al., First results with the novel petawatt laser acceleration facility in Dresden, J. Phys. 874, 012028 (2017).

[72] J. Couperus, A. Köhler, T. Wolterink, A. Jochmann, O. Zarini, H. Bastiaens, K. Boller, A. Irman, and U. Schramm,
Tomographic characterisation of gas-jet targets for laser wakefield acceleration, Nucl. Instrum. Methods Phys. Res., Sect. A 830, 504 (2016).

[73] A. Irman, J. P. Couperus, A. Debus, A. Köhler, J. M. Krämer, R. Pausch, O. Zarini, and U. Schramm, Improved performance of laser wakefield acceleration by tailored self-truncated ionization injection, Plasma Phys. Controlled Fusion 60, 044015 (2018).

[74] J. R. Fienup, Phase retrieval algorithms: A comparison Appl. Opt. 21, 2758 (1982).

[75] S. Marchesini, H. He, H. N. Chapman, S. P. Hau-Riege, A. Noy, M. R. Howells, U. Weierstall, and J.C.H. Spence, X-ray image reconstruction from a diffraction pattern alone, Phys. Rev. B 68, 140101(R) (2003). 